\title{
A New Type of Combination Synchronization among Multiple Chaotic Systems
}

\author{
Xingxu Wang $\left(\mathbb{D},{ }^{1}\right.$ Lin Sun $\mathbb{D}^{1},{ }^{1}$ Bingji Wang $\left(\mathbb{D},{ }^{2}\right.$ and Tousheng Huang $\mathbb{D}^{2}$ \\ ${ }^{1}$ School of Mechanical Engineering, Liaoning Shihua University, Fushun 113001, China \\ ${ }^{2}$ Research Center for Engineering Ecology and Nonlinear Science, North China Electric Power University, Beijing 102206, China \\ Correspondence should be addressed to Lin Sun; sunlin0626321@163.com
}

Received 11 January 2019; Revised 20 April 2019; Accepted 8 May 2019; Published 23 May 2019

Academic Editor: Rosa M. Benito

Copyright (c) 2019 Xingxu Wang et al. This is an open access article distributed under the Creative Commons Attribution License, which permits unrestricted use, distribution, and reproduction in any medium, provided the original work is properly cited.

\begin{abstract}
Based on former combination synchronization studies, a new type of combination synchronization approach is developed in this research, with the consideration of parallel combination of drive systems. This new synchronization approach is referred to as combination synchronization-II. As a representative case, the combination synchronization-II between three drive systems and one response system is studied. Applying Lyapunov stability theorem and active backstepping design, sufficient conditions for the proposed combination synchronization approach are derived. Numerical simulations are performed to show the feasibility and effectiveness of the proposed approach. Based on the investigation in this research, the proposed approach provides an applicable method for designing universal combination synchronization among multiple chaotic systems.
\end{abstract}

\section{Introduction}

Chaos phenomena have been widely observed in many systems and chaos synchronization plays an important role in the research on chaos. Great efforts were devoted to chaos synchronization of chaotic systems, a very important subfield of nonlinear science [1]. Since the pioneering work of Pecora and Carroll [2], chaos synchronization has been investigated in a variety of fields, such as ecological systems, chemical reactions, engineering science, and secure communication [3-5].

Many research works have been made to achieve various types of chaos synchronization, for example, complete synchronization [6,7], phase synchronization $[8,9]$, and generalized synchronization $[10,11]$. Nevertheless, most of the research works of chaos synchronization are limited to the synchronization of two chaotic systems, i.e., one drive system and one response system. In 2012, Luo et al. [12] developed a new type of synchronization, combination synchronization, which was applied to investigate the synchronization of two drive systems and one response system. From then on, the synchronization among multiple chaotic systems was extensively studied by many researchers [13-18].
The combination synchronization in recent literature develops in various types. Xi et al. proposed the function projective combination synchronization and determined the stability criterion for such combination synchronization of fractional-order chaotic systems [19]. Wang et al. investigated a novel adaptive generalized combination complex synchronization for different real and complex nonlinear systems with unknown parameters [20]. Vincent et al. developed a multiswitching combination synchronization of chaotic systems [18], and this synchronization type is further developed by Ahmad et al. as globally exponential multi-switchingcombination synchronization control for chaotic systems in the field of secure communications [21]. Based on the combination synchronization, with the consideration of four or more chaotic systems, the researchers further proposed and explored combination-combination synchronization in the cases where the numbers of drive systems and response systems are both larger than one [13, 22-25].

The application of combination synchronization has been widespread for different chaotic systems as recorded in literature. The chaos and combination synchronization of a new fractional-order Lorenz-like system with two stable node-foci was thoroughly studied by Alam et al. [26]. Khan and Shikha 


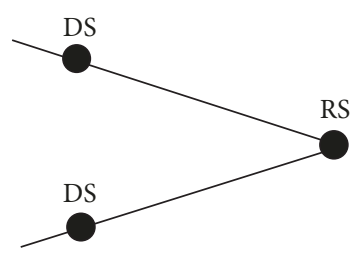

(a)

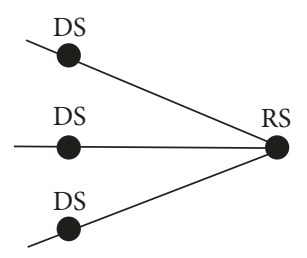

(b)

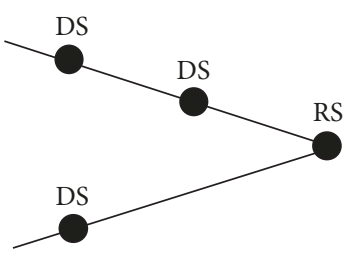

(c)

FIgURE 1: The parallel combination of two and three drive systems. DS: drive system; RS: response system.

used robust adaptive sliding mode control to investigate the combination synchronization of identical Genesio time-delay chaotic system [27]. Moreover, the combination synchronization scheme has also been introduced for complex-variable chaotic systems [17]. Sun et al. launched investigations on the finite-time combination synchronization of complex-variable chaotic systems with unknown parameters via sliding mode control $[28,29]$. Singh et al. proposed a novel scheme for the dual combination synchronization among four master systems and two slave systems for the fractional-order complex chaotic systems with stability analysis [30].

As so far, the combination synchronization approaches focus on series combination of drive systems; i.e., the drive systems can be regarded as one combined supersystem to synchronize with the response system. Actually, there are two basic ways for combination of three systems, series combination $(\bullet-\bullet-\bullet)$ and parallel combination $(\bullet \bullet)$. Based on the two combination ways, many complex systems can be composed, such as electric circuit and ecological food web $[31,32]$. The combination synchronization based on parallel combination of drive systems with one response system is described as the following: (1) the drive systems are divided into at least two groups, and each group exists as one of the parallel branches in the system combination; (2) the drive systems on the same branch jointly drive the response system; (3) the drive systems on different branches synchronize with the response system separately; and (4) the response system takes merely one controller in the combination synchronization. In this research, we refer the combination synchronization based on such parallel combination of drive systems as combination synchronizationII, whereas we refer to the former Runzi's combination synchronization as combination synchronization-I [12].

In comparing with former research works on the combination synchronization, the combination synchronizationII designed in this research may be a similar but extended type to combination complex synchronization or dual combination synchronization. The combination complex synchronization, for example, as described in the research of Sun et al. [17, 28, 29], suggests that the real part and the imaginary part of the drive systems and the response system, which are all complex systems, achieve combination synchronization, respectively. In this sense, the combination synchronizationII also considers that the synchronization of the chaotic systems can be divided into a few parallel branches or parts, similar to the division of real and imaginary parts in combination complex synchronization.
However, the combination synchronization-II exhibits three aspects of difference from the combination complex synchronization. Firstly, the response system commonly exists in every parallel branch divided for the synchronization. Secondly, the division of parallel branches for synchronization relies on the relationship between the chaotic systems in reality, and this is very different from the relation of real and imaginary parts in complex systems. Thirdly, according to the number of systems in each branch, synchronization between two chaotic systems or combination synchronization among three or more chaotic systems is achieved, implying that the synchronization in different braches can be unidentical. Based on the comparison with previous approaches in literature, the combination synchronizationII may be more general than the combination complex synchronization or the combination synchronization-I and it shows advantages in practical applications for more system synchronization modes. Therefore, the research on the combination synchronization-II still remains open and deserves investigation.

The organization of this research is organized as follows. Section 2 shows the scheme of combination synchronizationII with three drive systems and one response system, which shows two cases. In Section 3, we study the first case of the combination synchronization-II among four chaotic systems. Section 4 focuses on the achievement of the second case of the combination synchronization-II. At last, conclusions are described in Section 5.

\section{The Scheme of Combination Synchronization-II}

The combination synchronization-II is a drive-response type synchronization. Consider multiple drive systems and one response system, the parallel combination for drive systems may have various cases. It is easy to identify that the parallel combination of two drive systems has a simple structure as Figure 1(a). For three drive systems, there exist two cases for the parallel combination of drive systems, as shown in Figures 1(b) and 1(c). The case of Figure 1(b) describes three parallel branches, each one of which has merely one drive system whereas the case of Figure 1(c) demonstrates two branches, one of which has two serial drive systems. It should be noticed that the parallel combination of two drive systems can be simplified from the two cases of Figure 1(b) or Figure 1(c). Moreover, more complex cases of parallel combinations can be extended similarly from these two cases. 
Based on the above description, the two cases of Figures 1(b) and 1(c) can be regarded as representative cases. Therefore, we focus on the combination synchronization-II of three drive systems and one response system. In this section, the combination synchronization-II scheme for three drive systems and one response system is designed. The three drive systems are given as follows:

$$
\dot{x}^{(i)}=f^{(i)}\left(x^{(i)}\right) \quad(i=1,2,3),
$$

and the response system is given by

$$
\dot{z}=h(z)+u\left(x^{(1)}, x^{(2)}, x^{(3)}, z\right) .
$$

In the above equations, the symbols have the following meaning: $x^{(i)}=\left(x_{1}^{(i)}, x_{2}^{(i)}, \ldots, x_{n}^{(i)}\right)^{T}(i=1,2,3), z=$ $\left(z_{1}, z_{2}, \ldots, z_{n}\right)^{T} \in R^{n}$ are the state vectors of systems (1) and (2), respectively; $f^{(i)}(i=1,2,3), h: R^{n} \longrightarrow R^{n}$ are four continuous vector functions; $u=R^{n} \times R^{n} \times R^{n} \times R^{n} \longrightarrow R^{n}$ is a controller to be designed.

Definition 1. Combination synchronization-II between the drive systems (1) and the response system (2) is achieved if there exist constant matrixes $A_{i}, B_{j} \in R^{n}(i=1,2,3 ; j=$ $1,2, \cdots, l)$ and $B_{j} \neq 0$ such that

$$
\sum_{j=1}^{l} \lim _{t \rightarrow+\infty}\left\|\sum_{i=k_{j-1}+1}^{k_{j}} A_{i} x^{(i)}-B_{j} z\right\|=0
$$

in which $\|\cdot\|$ represents the matrix norm; $l$ and $k_{j}(j=$ $0,1,2, \cdots, l)$ are integers and satisfy $1<l \leq 3$ and $0=k_{0}<$ $k_{1}<\cdots<k_{l}=3$.

Remark 2. The constant matrixes $A_{i}(i=1,2,3), B_{j}(j=$ $1,2, \cdots, l)$ are called scaling matrixes. Moreover, these matrixes can be extended to functional matrixes of the state variables $x^{(i)}(i=1,2,3)$ and $z$.

Remark 3. If (1) $l=1$; or (2) $l=2, k_{1}=1, A_{1}=B_{1}=0$; or (3) $l=2, k_{1}=2, A_{3}=B_{2}=0$, the combination synchronization-II problem will be turned into the combination synchronization-I problem.

Remark 4. If (1) $l=2, k_{1}=1$, (1i) $A_{1}=0$ or (1ii) $A_{2}=0$; or (2) $l=2, k_{1}=2$, (2i) $A_{2}=0$ or (2ii) $A_{3}=0$; or (3) $l=3$, $k_{1}=1, k_{2}=2$, (3i) $A_{1}=B_{1}=0$ or (3ii) $A_{2}=B_{2}=0$ or (3iii) $A_{3}=B_{3}=0$, the combination synchronization-II problem between three drive systems and one response system will be reduced to the combination synchronization-II problem between two drive systems and one response system.

Remark 5. If (1) $l=1, B_{1}=I$, (1i) $A_{1}=A_{2}=0$ or (lii) $A_{1}=A_{3}=0$ or (1iii) $A_{2}=A_{3}=0$; or (2) $l=2, k_{1}=1$, (2i) $B_{1}=I$ and $A_{2}=A_{3}=B_{2}=0$ or (2ii) $B_{2}=I$ and $A_{1}=A_{2}=B_{1}=0$ or (2iii) $B_{2}=I$ and $A_{1}=A_{3}=B_{1}=0$; or (3) $l=2, k_{1}=2$, (3i) $B_{1}=I$ and $A_{1}=A_{3}=B_{2}=0$ or (3ii) $B_{1}=I$ and $A_{2}=A_{3}=B_{2}=0$ or (3iii) $B_{2}=I$ and $A_{1}=A_{2}=B_{1}=0$; or (4) $l=3, k_{1}=1, k_{2}=2$, (4i) $B_{1}=I$ and $A_{2}=A_{3}=B_{2}=B_{3}=0$ or (4ii) $B_{2}=I$ and $A_{1}=A_{3}=$ $B_{1}=B_{3}=0$ or (4iii) $B_{3}=I$ and $A_{1}=A_{2}=B_{1}=B_{2}=0$, the combination synchronization-II problem will be reduced to the projective synchronization, where $I$ is an $n \times n$ identity matrix.

Remark 6. If (1) $l=1, A_{1}=A_{2}=A_{3}=0$; or (2) $l=2$, $k_{1}=1$ or $2, A_{1}=A_{2}=A_{3}=0$, (2i) $B_{1}=0$ or (2ii) $B_{2}=$ 0 ; or (3) $l=3, k_{1}=1, k_{2}=2, A_{1}=A_{2}=A_{3}=0$, (3i) $B_{1}=B_{2}=0$ or (3ii) $B_{1}=B_{3}=0$ or (3iii) $B_{2}=B_{3}=0$, the combination synchronization-II problem will be turned into a chaos control problem.

Remark 7. The combination synchronization-II scheme as described in Definition 1 can be extended to the cases which have more than three drive systems.

According to Definition 1, $l$ can have two values, 2 and 3, for the combination synchronization-II between three drive systems and one response system. In this research, we define the case of $l=3$ as Case 1 , and the case of $l=2$ as Case 2, corresponding to the two cases of Figures 1(b) and 1(c). The applications of the combination synchronization-II scheme for the two cases are provided in the following.

\section{Combination Synchronization-II of Case 1}

The combination synchronization-II scheme is applied to four chaotic systems. Such application can adapt to a variety of chaotic systems in engineering science, secure communication, etc. As widely known, Lorenz system, Chen system, Rössler system, and Lü system are four basic and classical chaotic systems, which have been studied by many researchers [33-35] and can be extended for designing combination synchronization-II scheme. Also, the memristor chaotic systems, for example, are also suitable cases for the investigation of combination synchronization-II. Recently, autonomous memristor systems of infinite chaotic attractors were proposed and electronic circuits of such memristor chaotic systems have been designed [36, 37]. With circuitry realization where memristor chaotic systems show parallel combination, the combination synchronization-II scheme can be extended for investigating multiple memristor systems of infinite chaotic attractors $[36,37]$.

In this section, the combination synchronization-II of Case 1 is realized among the four classic chaotic systems, Lorenz system, Chen system, Rössler system, and Lü system. We consider the first three systems as drive systems and Lü system as response system. The Lorenz system, Chen system, and Rössler system are described by the following systems (4), (5), and (6), respectively,

$$
\begin{aligned}
& \dot{x}_{1}=a_{1}\left(x_{2}-x_{1}\right), \\
& \dot{x}_{2}=b_{1} x_{1}-x_{2}-x_{1} x_{3}, \\
& \dot{x}_{3}=x_{1} x_{2}-c_{1} x_{3},
\end{aligned}
$$




$$
\begin{aligned}
& \dot{y}_{1}=a_{2}\left(y_{2}-y_{1}\right), \\
& \dot{y}_{2}=\left(c_{2}-a_{2}\right) y_{1}+c_{2} y_{2}-y_{1} y_{3}, \\
& \dot{y}_{3}=y_{1} y_{2}-b_{2} y_{3} . \\
& \dot{w}_{1}=-w_{2}-w_{3}, \\
& \dot{w}_{2}=w_{1}+a_{3} w_{2}, \\
& \dot{w}_{3}=b_{3}+w_{3}\left(w_{1}-c_{3}\right),
\end{aligned}
$$

As described in literature [12, 13], Lorenz system shows chaotic behavior when $a_{1}=10, b_{1}=28$, and $c_{1}=8 / 3$; Chen system exhibits chaotic behavior when $a_{2}=35, b_{2}=3$, and $c_{2}=28$; Rössler system exhibits chaotic behavior when $a_{3}=0.2, b_{3}=0.2$, and $c_{3}=5.7$. On the other hand, the controlled Lü system is given by

$$
\begin{aligned}
& \dot{z}_{1}=a_{4}\left(z_{2}-z_{1}\right)+u_{1}, \\
& \dot{z}_{2}=c_{4} z_{2}-z_{1} z_{3}+u_{2}, \\
& \dot{z}_{3}=z_{1} z_{2}-b_{4} z_{3}+u_{3} .
\end{aligned}
$$

Lü system exhibits chaotic behavior when $a_{4}=36, b_{4}=3$, and $c_{4}=20 . \operatorname{In}(7), u_{1}, u_{2}$, and $u_{3}$ are controllers to be designed.

Notice that to avoid confusions in the calculations, the state variables of the three drive systems in Definition 1 are redenoted as $x=\left(x_{1}, x_{2}, x_{3}\right)^{T}, y=\left(y_{1}, y_{2}, y_{3}\right)^{T}$ and $w=\left(w_{1}, w_{2}, w_{3}\right)^{T}$ in (4), (5), and (6). Simultaneously, the constant matrixes in Definition 1 are also redenoted as $A, B$, $C, D, E$, and $H$. According to Definition 1, the combination synchronization-II of Case 1 can be redescribed by

$$
\begin{gathered}
\lim _{t \rightarrow+\infty}\|A x-B z\|+\lim _{t \longrightarrow+\infty}\|C y-D z\| \\
+\lim _{t \longrightarrow+\infty}\|E w-H z\|=0 .
\end{gathered}
$$

For convenience of the calculations, we write the constant matrixes $A, B, C, D, E$, and $H$ as $A=\operatorname{diag}\left(\alpha_{1}, \alpha_{2}, \alpha_{3}\right), B=$ $\operatorname{diag}\left(\beta_{1}, \beta_{2}, \beta_{3}\right), C=\operatorname{diag}\left(\gamma_{1}, \gamma_{2}, \gamma_{3}\right), D=\operatorname{diag}\left(\delta_{1}, \delta_{2}, \delta_{3}\right), E=$ $\operatorname{diag}\left(\varepsilon_{1}, \varepsilon_{2}, \varepsilon_{3}\right)$, and $H=\operatorname{diag}\left(\eta_{1}, \eta_{2}, \eta_{3}\right)$, and let

$$
\begin{aligned}
& e_{1}=B z-A x, \\
& e_{2}=D z-C y, \\
& e_{3}=H z-E w,
\end{aligned}
$$

in which $e_{1}=\left(e_{11}, e_{12}, e_{13}\right)^{T}, e_{2}=\left(e_{21}, e_{22}, e_{23}\right)^{T}$, and $e_{3}=$ $\left(e_{31}, e_{32}, e_{33}\right)^{T}$. Hence, the error system can be written as the following:

$$
\begin{aligned}
\dot{e}_{11}= & \frac{a_{4} \beta_{1}}{\beta_{2}} e_{12}-a_{4} e_{11}+f_{1}+\beta_{1} u_{1}, \\
\dot{e}_{12}= & c_{4} e_{12}-\frac{\beta_{2}}{\beta_{1} \beta_{3}} e_{11} e_{13}-\frac{\alpha_{3} \beta_{2}}{\beta_{1} \beta_{3}} x_{3} e_{11}-\frac{\alpha_{1} \beta_{2}}{\beta_{1} \beta_{3}} x_{1} e_{13} \\
& +g_{1}+\beta_{2} u_{2},
\end{aligned}
$$

$$
\begin{aligned}
& \dot{e}_{13}=\frac{\beta_{3}}{\beta_{1} \beta_{2}} e_{11} e_{12}+\frac{\alpha_{2} \beta_{3}}{\beta_{1} \beta_{2}} x_{2} e_{11}+\frac{\alpha_{1} \beta_{3}}{\beta_{1} \beta_{2}} x_{1} e_{12}-b_{4} e_{13} \\
& +h_{1}+\beta_{3} u_{3} \text {, } \\
& \dot{e}_{21}=\frac{a_{4} \delta_{1}}{\delta_{2}} e_{22}-a_{4} e_{21}+f_{2}+\delta_{1} u_{1} \text {, } \\
& \dot{e}_{22}=c_{4} e_{22}-\frac{\delta_{2}}{\delta_{1} \delta_{3}} e_{21} e_{23}-\frac{\gamma_{3} \delta_{2}}{\delta_{1} \delta_{3}} y_{3} e_{21}-\frac{\gamma_{1} \delta_{2}}{\delta_{1} \delta_{3}} y_{1} e_{23} \\
& +g_{2}+\delta_{2} u_{2} \\
& \dot{e}_{23}=\frac{\delta_{3}}{\delta_{1} \delta_{2}} e_{21} e_{22}+\frac{\gamma_{2} \delta_{3}}{\delta_{1} \delta_{2}} y_{2} e_{21}+\frac{\gamma_{1} \delta_{3}}{\delta_{1} \delta_{2}} y_{1} e_{22}-b_{4} e_{23} \\
& +h_{2}+\delta_{3} u_{3} \\
& \dot{e}_{31}=\frac{a_{4} \eta_{1}}{\eta_{2}} e_{32}-a_{4} e_{31}+f_{3}+\eta_{1} u_{1} \text {, } \\
& \dot{e}_{32}=c_{4} e_{32}-\frac{\eta_{2}}{\eta_{1} \eta_{3}} e_{31} e_{33}-\frac{\varepsilon_{3} \eta_{2}}{\eta_{1} \eta_{3}} w_{3} e_{31}-\frac{\varepsilon_{1} \eta_{2}}{\eta_{1} \eta_{3}} w_{1} e_{33} \\
& +g_{3}+\eta_{2} u_{2} \\
& \dot{e}_{33}=\frac{\eta_{3}}{\eta_{1} \eta_{2}} e_{31} e_{32}+\frac{\varepsilon_{2} \eta_{3}}{\eta_{1} \eta_{2}} w_{2} e_{31}+\frac{\varepsilon_{1} \eta_{3}}{\eta_{1} \eta_{2}} w_{1} e_{32}-b_{4} e_{33} \\
& +h_{3}+\eta_{3} u_{3}
\end{aligned}
$$

in which

$$
\begin{aligned}
f_{1}= & \frac{a_{4} \alpha_{2} \beta_{1}}{\beta_{2}} x_{2}-a_{4} \alpha_{1} x_{1}-a_{1} \alpha_{1}\left(x_{2}-x_{1}\right), \\
g_{1}= & \left(c_{4}+1\right) \alpha_{2} x_{2}-b_{1} \alpha_{2} x_{1}+\left(\alpha_{2}-\frac{\alpha_{1} \alpha_{3} \beta_{2}}{\beta_{1} \beta_{3}}\right) x_{1} x_{3}, \\
h_{1}= & \left(\frac{\alpha_{1} \alpha_{2} \beta_{3}}{\beta_{1} \beta_{2}}-\alpha_{3}\right) x_{1} x_{2}+\left(c_{1}-b_{4}\right) \alpha_{3} x_{3}, \\
f_{2}= & \frac{a_{4} \gamma_{2} \delta_{1}}{\delta_{2}} y_{2}-a_{4} \gamma_{1} y_{1}-a_{2} \gamma_{1}\left(y_{2}-y_{1}\right), \\
g_{2}= & \left(c_{4}-c_{2}\right) \gamma_{2} y_{2}-\left(c_{2}-a_{2}\right) \gamma_{2} y_{1} \\
& +\left(\gamma_{2}-\frac{\gamma_{1} \gamma_{3} \delta_{2}}{\delta_{1} \delta_{3}}\right) y_{1} y_{3}, \\
h_{2}= & \left(\frac{\gamma_{1} \gamma_{2} \delta_{3}}{\delta_{1} \delta_{2}}-\gamma_{3}\right) y_{1} y_{2}+\left(b_{2}-b_{4}\right) \gamma_{3} y_{3}, \\
f_{3}= & -a_{4} \varepsilon_{1} w_{1}+\left(\frac{a_{4} \varepsilon_{2} \eta_{1}}{\eta_{2}}+\varepsilon_{1}\right) w_{2}+\varepsilon_{1} w_{3}, \\
g_{3}= & -\frac{\varepsilon_{1} \varepsilon_{3} \eta_{2}}{\eta_{1} \eta_{3}} w_{1} w_{3}-\varepsilon_{2} w_{1}+\left(c_{4}-a_{3}\right) \varepsilon_{2} w_{2}, \\
h_{3}= & \frac{\varepsilon_{1} \varepsilon_{2} \eta_{3}}{\eta_{1} \eta_{2}} w_{1} w_{2}-\varepsilon_{3} w_{1} w_{3}+\left(c_{3}-b_{4}\right) \varepsilon_{3} w_{3}-b_{3} \varepsilon_{3} .
\end{aligned}
$$

Based on the active backstepping design method, the controllers $u_{1}, u_{2}$, and $u_{3}$ can be designed in the following 
for realizing the combination synchronization-II of Case 1 among Lorenz system, Chen system, Rössler system, and Lü system. For the details of the active backstepping design method, one can refer to Luo et al. [12].

Theorem 8. If the controllers are chosen as

$$
\begin{aligned}
& u_{1}=-\frac{s_{1}}{\beta_{1}} f_{1}-\frac{s_{2}}{\delta_{1}} f_{2}-\frac{s_{3}}{\eta_{1}} f_{3}, \\
& u_{2}=-s_{1}\left(\frac{\left(a_{4}-1\right)\left(c_{4}+1\right)}{a_{4} \beta_{1}}+\frac{a_{4} \beta_{1}}{\beta_{2}^{2}}-\frac{\alpha_{3} x_{3}}{\beta_{1} \beta_{3}}\right) v_{11} \\
& -s_{2}\left(\frac{\left(a_{4}-1\right)\left(c_{4}+1\right)}{a_{4} \delta_{1}}+\frac{a_{4} \delta_{1}}{\delta_{2}^{2}}-\frac{\gamma_{3} y_{3}}{\delta_{1} \delta_{3}}\right) v_{21} \\
& -s_{3}\left(\frac{\left(a_{4}-1\right)\left(c_{4}+1\right)}{a_{4} \eta_{1}}+\frac{a_{4} \eta_{1}}{\eta_{2}^{2}}-\frac{\varepsilon_{3} w_{3}}{\eta_{1} \eta_{3}}\right) v_{31}-\left(c_{4}\right. \\
& \left.-a_{4}+2\right)\left(\frac{s_{1}}{\beta_{2}} v_{12}+\frac{s_{2}}{\delta_{2}} v_{22}+\frac{s_{3}}{\eta_{2}} v_{32}\right)-\frac{s_{1}}{\beta_{2}} g_{1}-\frac{s_{2}}{\delta_{2}} \\
& \cdot g_{2}-\frac{s_{3}}{\eta_{2}} g_{3} \\
& u_{3}=-s_{1}\left[\left(\frac{a_{4}-1}{a_{4} \beta_{1}^{2}} v_{11}+\frac{1}{\beta_{1}}\left(\frac{1}{\beta_{2}}-\frac{\beta_{2}}{\beta_{3}^{2}}\right) v_{12}\right)\right. \\
& \left.\cdot\left(v_{11}+\alpha_{1} x_{1}\right)+\frac{\alpha_{2}}{\beta_{1} \beta_{2}} x_{2} v_{11}\right] \\
& -s_{2}\left[\left(\frac{a_{4}-1}{a_{4} \delta_{1}^{2}} v_{21}+\frac{1}{\delta_{1}}\left(\frac{1}{\delta_{2}}-\frac{\delta_{2}}{\delta_{3}^{2}}\right) v_{22}\right)\right. \\
& \left.\cdot\left(v_{21}+\gamma_{1} y_{1}\right)+\frac{\gamma_{2}}{\delta_{1} \delta_{2}} y_{2} v_{21}\right] \\
& -s_{3}\left[\left(\frac{a_{4}-1}{a_{4} \eta_{1}^{2}} v_{31}+\frac{1}{\eta_{1}}\left(\frac{1}{\eta_{2}}-\frac{\eta_{2}}{\eta_{3}^{2}}\right) v_{32}\right)\right. \\
& \left.\cdot\left(v_{31}+\varepsilon_{1} w_{1}\right)+\frac{\varepsilon_{2}}{\eta_{1} \eta_{2}} w_{2} v_{31}\right]+\left(b_{4}-1\right)\left(\frac{s_{1}}{\beta_{3}} v_{13}\right. \\
& \left.+\frac{s_{2}}{\delta_{3}} v_{23}+\frac{s_{3}}{\eta_{3}} v_{33}\right)-\frac{s_{1}}{\beta_{3}} h_{1}-\frac{s_{2}}{\delta_{3}} h_{2}-\frac{s_{3}}{\eta_{3}} h_{3} \text {, }
\end{aligned}
$$

in which

$$
\begin{aligned}
s_{1} & =\operatorname{sgn}(\|x\|)(1-\operatorname{sgn}(\|y\|))(1-\operatorname{sgn}(\|w\|)), \\
s_{2} & =\operatorname{sgn}(\|y\|)(1-\operatorname{sgn}(\|x\|))(1-\operatorname{sgn}(\|w\|)), \\
s_{3} & =\operatorname{sgn}(\|w\|)(1-\operatorname{sgn}(\|x\|))(1-\operatorname{sgn}(\|y\|)), \\
\left(\begin{array}{c}
v_{11} \\
v_{12} \\
v_{13}
\end{array}\right) & =\left(\begin{array}{ccc}
1 & 0 & 0 \\
\frac{\left(1-a_{4}\right) \beta_{2}}{a_{4} \beta_{1}} & 1 & 0 \\
0 & 0 & 1
\end{array}\right)\left(\begin{array}{l}
e_{11} \\
e_{12} \\
e_{13}
\end{array}\right),
\end{aligned}
$$

$$
\begin{aligned}
& \left(\begin{array}{l}
v_{21} \\
v_{22} \\
v_{23}
\end{array}\right)=\left(\begin{array}{ccc}
1 & 0 & 0 \\
\frac{\left(1-a_{4}\right) \delta_{2}}{a_{4} \delta_{1}} & 1 & 0 \\
0 & 0 & 1
\end{array}\right)\left(\begin{array}{l}
e_{21} \\
e_{22} \\
e_{23}
\end{array}\right), \\
& \left(\begin{array}{l}
v_{31} \\
v_{32} \\
v_{33}
\end{array}\right)=\left(\begin{array}{ccc}
1 & 0 & 0 \\
\frac{\left(1-a_{4}\right) \eta_{2}}{a_{4} \eta_{1}} & 1 & 0 \\
0 & 0 & 1
\end{array}\right)\left(\begin{array}{l}
e_{31} \\
e_{32} \\
e_{33}
\end{array}\right),
\end{aligned}
$$

the drive systems (4), (5), and (6) will achieve combination synchronization-II (Case 1) with the response system (7).

Proof. Based on the linear transformation described in (14), we construct $\left(v_{11}, v_{12}, v_{13}\right)$-subsystem. According to the definition of combination synchronization-II, we have $\|y\|=0$ and $\|w\|=0$ for this subsystem. Then direct calculations lead to

$$
\begin{aligned}
\dot{v}_{11}= & \frac{a_{4} \beta_{1}}{\beta_{2}}\left(v_{12}-\frac{\left(1-a_{4}\right) \beta_{2}}{a_{4} \beta_{1}} v_{11}\right)-a_{4} v_{11}+f_{1} \\
& +\left.\beta_{1} u_{1}\right|_{\|x\|>0,\|y\|=0,\|w\|=0}=-v_{11}+\frac{a_{4} \beta_{1}}{\beta_{2}} v_{12}, \\
\dot{v}_{12}= & \frac{\left(1-a_{4}\right) \beta_{2}}{a_{4} \beta_{1}}\left(-v_{11}+\frac{a_{4} \beta_{1}}{\beta_{2}} v_{12}\right) \\
& +c_{4}\left(v_{12}-\frac{\left(1-a_{4}\right) \beta_{2}}{a_{4} \beta_{1}} v_{11}\right)-\frac{\beta_{2}}{\beta_{1} \beta_{3}} v_{11} v_{13} \\
& -\frac{\alpha_{3} \beta_{2}}{\beta_{1} \beta_{3}} x_{3} v_{11}-\frac{\alpha_{1} \beta_{2}}{\beta_{1} \beta_{3}} x_{1} v_{13}+g_{1} \\
& +\left.\beta_{2} u_{2}\right|_{\|x\|>0,\|y\|=0,\|w\|=0} \\
= & -\frac{a_{4} \beta_{1}}{\beta_{2}} v_{11}-v_{12}-\frac{\beta_{2}}{\beta_{1} \beta_{3}}\left(v_{11}+\alpha_{1} x_{1}\right) v_{13}, \\
\dot{v}_{13}= & \left.\frac{\beta_{3}}{\beta_{1} \beta_{2}} v_{11}+\frac{\alpha_{1} \beta_{3}}{\beta_{1} \beta_{2}} x_{1}\right)\left(v_{12}-\frac{\left(1-a_{4}\right) \beta_{2}}{a_{4} \beta_{1}} v_{11}\right) \\
& \left.+\frac{\alpha_{2} \beta_{3}}{\beta_{1} \beta_{2}} x_{2} v_{11}-b_{4} v_{13}+h_{1}+\alpha_{1} x_{1}\right) v_{12}-v_{13} . \\
& +\left.\beta_{3} u_{3}\right|_{\|x\|>0,\|y\|=0,\|w\|=0} \\
&
\end{aligned}
$$

We can take a Lyapunov function as

$$
V_{1}=\frac{1}{2} v_{11}^{2}+\frac{1}{2} v_{12}^{2}+\frac{1}{2} v_{13}^{2}
$$


for the $\left(v_{11}, v_{12}, v_{13}\right)$-subsystem. The derivative of $V_{1}$ is given by

$$
\begin{aligned}
\dot{V}_{1}= & v_{11} \dot{v}_{11}+v_{12} \dot{v}_{12}+v_{13} \dot{v}_{13} \\
= & v_{11}\left(-v_{11}+\frac{a_{4} \beta_{1}}{\beta_{2}} v_{12}\right) \\
& +v_{12}\left(-\frac{a_{4} \beta_{1}}{\beta_{2}} v_{11}-v_{12}-\frac{\beta_{2}}{\beta_{1} \beta_{3}}\left(v_{11}+\alpha_{1} x_{1}\right) v_{13}\right) \\
& +v_{13}\left(\frac{\beta_{2}}{\beta_{1} \beta_{3}}\left(v_{11}+\alpha_{1} x_{1}\right) v_{12}-v_{13}\right) \\
= & -v_{11}^{2}-v_{12}^{2}-v_{13}^{2} \leq 0 .
\end{aligned}
$$

According to the Lyapunov stability theorem, $\dot{V}_{1} \leq 0$ as shown in (17) suggests that the equilibrium $(0,0,0)$ is globally asymptotically stable for the $\left(v_{11}, v_{12}, v_{13}\right)$-subsystem. Likewise, we construct $\left(v_{21}, v_{22}, v_{23}\right)$-subsystem and we have $\|x\|=$ 0 and $\|w\|=0$ for this subsystem. Meanwhile, the following can be obtained:

$$
\begin{aligned}
\dot{v}_{21}= & \frac{a_{4} \delta_{1}}{\delta_{2}}\left(v_{22}-\frac{\left(1-a_{4}\right) \delta_{2}}{a_{4} \delta_{1}} v_{21}\right)-a_{4} v_{21}+f_{2} \\
& +\left.\delta_{1} u_{1}\right|_{\|x\|=0,\|y\|>0,\|w\|=0}=-v_{21}+\frac{a_{4} \delta_{1}}{\delta_{2}} v_{22}, \\
\dot{v}_{22}= & \frac{\left(1-a_{4}\right) \delta_{2}}{a_{4} \delta_{1}}\left(-v_{21}+\frac{a_{4} \delta_{1}}{\delta_{2}} v_{22}\right) \\
& +c_{4}\left(v_{22}-\frac{\left(1-a_{4}\right) \delta_{2}}{a_{4} \delta_{1}} v_{21}\right)-\frac{\delta_{2}}{\delta_{1} \delta_{3}} v_{21} v_{23} \\
& -\frac{\gamma_{3} \delta_{2}}{\delta_{1} \delta_{3}} y_{3} v_{21}-\frac{\gamma_{1} \delta_{2}}{\delta_{1} \delta_{3}} y_{1} v_{23}+g_{2} \\
& +\left.\delta_{2} u_{2}\right|_{\|x\|=0,\|y\|>0,\|w\|=0} \\
= & -\frac{a_{4} \delta_{1}}{\delta_{2}} v_{21}-v_{22}-\frac{\delta_{2}}{\delta_{1} \delta_{3}}\left(v_{21}+\gamma_{1} y_{1}\right) v_{23}, \\
\dot{v}_{23}= & \left(\frac{\delta_{3}}{\delta_{1} \delta_{2}} v_{21}+\frac{\gamma_{1} \delta_{3}}{\delta_{1} \delta_{2}} y_{1}\right)\left(v_{22}-\frac{\left(1-a_{4}\right) \delta_{2}}{a_{4} \delta_{1}} v_{21}\right) \\
& +\frac{\gamma_{2} \delta_{3}}{\delta_{1} \delta_{2}} y_{2} v_{21}-b_{4} v_{23}+h_{2} \\
& +\left.\delta_{3} u_{3}\right|_{\|x\|=0,\|y\|>0,\|w\|=0} \\
= & \frac{\delta_{2}}{\delta_{1} \delta_{3}}\left(v_{21}+\gamma_{1} y_{1}\right) v_{22}-v_{23},
\end{aligned}
$$

Choosing a candidate Lyapunov function,

$$
V_{2}=\frac{1}{2} v_{21}^{2}+\frac{1}{2} v_{22}^{2}+\frac{1}{2} v_{23}^{2},
$$

for the $\left(v_{21}, v_{22}, v_{23}\right)$-subsystem. The derivative of $V_{2}$ is given by

$$
\begin{aligned}
\dot{V}_{2}= & v_{21} \dot{v}_{21}+v_{22} \dot{v}_{22}+v_{23} \dot{v}_{23} \\
= & v_{21}\left(-v_{21}+\frac{a_{4} \delta_{1}}{\delta_{2}} v_{22}\right) \\
& +v_{22}\left(-\frac{a_{4} \delta_{1}}{\delta_{2}} v_{21}-v_{22}-\frac{\delta_{2}}{\delta_{1} \delta_{3}}\left(v_{21}+\gamma_{1} y_{1}\right) v_{23}\right) \\
& +v_{23}\left(\frac{\delta_{2}}{\delta_{1} \delta_{3}}\left(v_{21}+\gamma_{1} y_{1}\right) v_{22}-v_{23}\right) \\
= & -v_{21}^{2}-v_{22}^{2}-v_{23}^{2} \leq 0,
\end{aligned}
$$

which suggests that $\left(v_{21}, v_{22}, v_{23}\right)$ asymptotically approaches $(0,0,0)$ as time increases to infinite.

Construct $\left(v_{31}, v_{32}, v_{33}\right)$-subsystem which satisfies $\|x\|=$ 0 and $\|y\|=0$. Then we have

$$
\begin{aligned}
\dot{v}_{31}= & \frac{a_{4} \eta_{1}}{\eta_{2}}\left(v_{32}+\frac{\left(a_{4}-1\right) \eta_{2}}{a_{4} \eta_{1}} v_{31}\right)-a_{4} v_{31}+f_{3} \\
& +\left.\eta_{1} u_{1}\right|_{\|x\|=0,\|y\|=0,\|w\|>0}=-v_{31}+\frac{a_{4} \eta_{1}}{\eta_{2}} v_{32}, \\
\dot{v}_{32}= & \frac{\left(1-a_{4}\right) \eta_{2}}{a_{4} \eta_{1}}\left(-v_{31}+\frac{a_{4} \eta_{1}}{\eta_{2}} v_{32}\right) \\
& +c_{4}\left(v_{32}+\frac{\left(a_{4}-1\right) \eta_{2}}{a_{4} \eta_{1}} v_{31}\right)-\frac{\eta_{2}}{\eta_{1} \eta_{3}} v_{31} v_{33} \\
& -\frac{\varepsilon_{3} \eta_{2}}{\eta_{1} \eta_{3}} w_{3} v_{31}-\frac{\varepsilon_{1} \eta_{2}}{\eta_{1} \eta_{3}} w_{1} v_{33}+g_{3} \\
& +\left.\eta_{2} u_{2}\right|_{\|x\|=0,\|y\|=0,\|w\|>0} \\
= & -\frac{a_{4} \eta_{1}}{\eta_{2}} v_{31}-v_{32}-\frac{\eta_{2}}{\eta_{1} \eta_{3}}\left(v_{31}+\varepsilon_{1} w_{1}\right) v_{33}, \\
\dot{v}_{33}= & \left.\frac{\eta_{3}}{\eta_{1} \eta_{2}} v_{31}+\frac{\varepsilon_{1} \eta_{3}}{\eta_{1} \eta_{2}} w_{1}\right)\left(v_{32}+\frac{\left(a_{4}-1\right) \eta_{2}}{a_{4} \eta_{1}} v_{31}\right) \\
& +\frac{\varepsilon_{2} \eta_{3}}{\eta_{1} \eta_{2}} w_{2} v_{31}-b_{4} v_{33}+h_{3} \\
& +\left.\eta_{3} u_{3}\right|_{\|x\|=0,\|y\|=0,\|w\|>0} \\
= & \frac{\eta_{2}}{\eta_{1} \eta_{3}}\left(v_{31}+\varepsilon_{1} w_{1}\right) v_{32}-v_{33} .
\end{aligned}
$$

Choosing a candidate Lyapunov function,

$$
V_{3}=\frac{1}{2} v_{31}^{2}+\frac{1}{2} v_{32}^{2}+\frac{1}{2} v_{33}^{2},
$$

for the $\left(v_{31}, v_{32}, v_{33}\right)$-subsystem. The derivative of $V_{3}$ is given by

$$
\begin{aligned}
\dot{V}_{3} & =v_{31} \dot{v}_{31}+v_{32} \dot{v}_{32}+v_{33} \dot{v}_{33} \\
& =v_{31}\left(-v_{31}+\frac{a_{4} \eta_{1}}{\eta_{2}} v_{32}\right)
\end{aligned}
$$




$$
\begin{aligned}
& +v_{32}\left(-\frac{a_{4} \eta_{1}}{\eta_{2}} v_{31}-v_{32}-\frac{\eta_{2}}{\eta_{1} \eta_{3}}\left(v_{31}+\varepsilon_{1} w_{1}\right) v_{33}\right) \\
& +v_{33}\left(\frac{\eta_{2}}{\eta_{1} \eta_{3}}\left(v_{31}+\varepsilon_{1} w_{1}\right) v_{32}-v_{33}\right) \\
& =-v_{31}^{2}-v_{32}^{2}-v_{33}^{2} \leq 0,
\end{aligned}
$$

which suggests that $\left(v_{31}, v_{32}, v_{33}\right)$ asymptotically approaches $(0,0,0)$.

Taking the above results together, we have $\left(v_{11}, v_{12}, v_{13}\right)$ $\longrightarrow(0,0,0),\left(v_{21}, v_{22}, v_{23}\right) \longrightarrow(0,0,0)$, and $\left(v_{31}, v_{32}, v_{33}\right) \longrightarrow$
$(0,0,0)$ as $t \rightarrow+\infty$. Notice the linear transformation described by (14), we know $e_{i j} \longrightarrow 0(i=1,2,3 ; j=$ $1,2,3)$. It means that the drive systems (4), (5), and (6) achieve combination synchronization-II with the response system (7).

From Theorem 8, the following corollaries can be deduced. Since the proofs of the corollaries are similar to Theorem 8, we omit the corollary proofs for the sake of simplification.

Corollary 9. If the controllers are chosen as

$$
u=\left(\begin{array}{l}
\frac{\left(\left.\beta_{1} u_{1}\right|_{\|x\|>0,\|y\|=0,\|w\|=0}+\left.\delta_{1} u_{1}\right|_{\|x\|=0,\|y\|>0,\|w\|=0}+\left.\eta_{1} u_{1}\right|_{\|x\|=0,\|y\|=0,\|w\|>0}\right)}{\left(\beta_{1}+\delta_{1}+\eta_{1}\right)} \\
\frac{\left(\left.\beta_{2} u_{2}\right|_{\|x\|>0,\|y\|=0,\|w\|=0}+\left.\delta_{2} u_{2}\right|_{\|x\|=0,\|y\|>0,\|w\|=0}+\left.\eta_{2} u_{2}\right|_{\|x\|=0,\|y\|=0,\|w\|>0}\right)}{\left(\beta_{2}+\delta_{2}+\eta_{2}\right)} \\
\frac{\left(\left.\beta_{3} u_{3}\right|_{\|x\|>0,\|y\|=0,\|w\|=0}+\left.\delta_{3} u_{3}\right|_{\|x\|=0,\|y\|>0,\|w\|=0}+\left.\eta_{3} u_{3}\right|_{\|x\|=0,\|y\|=0,\|w\|>0}\right)}{\left(\beta_{3}+\delta_{3}+\eta_{3}\right)}
\end{array}\right),
$$

in which $u_{1}, u_{2}$, and $u_{3}$ are described by (10), we will have

$$
\lim _{t \rightarrow+\infty}\|A x+C y+E w-(B+D+H) z\|=0 .
$$

Hence, the drive systems (4), (5), and (6) can achieve combination synchronization-I with the response system (7).

Corollary 10. (i) Let $\alpha_{1}=\alpha_{2}=\alpha_{3}=0$ and $\beta_{1}=$ $\beta_{2}=\beta_{3}=0$; if the controllers are chosen as (10) or (24) with removing all the terms related to $v_{11}, v_{12}, v_{13}$, the drive systems (5) and (6) can achieve combination synchronizationII or combination synchronization-I with the response system (7). (ii) Let $\gamma_{1}=\gamma_{2}=\gamma_{3}=0$ and $\delta_{1}=\delta_{2}=\delta_{3}=0$; if the controllers are chosen as (10) or (24) with removing all the terms related to $v_{21}, v_{22}, v_{23}$, the drive systems (4) and (6) can achieve combination synchronization-II or combination synchronization-I with the response system (7).

(iii) Let $\varepsilon_{1}=\varepsilon_{2}=\varepsilon_{3}=0$ and $\eta_{1}=\eta_{2}=\eta_{3}=0$; if the controllers are chosen as (10) or (24) with removing all the terms related to $v_{31}, v_{32}, v_{33}$, the drive systems (4) and (5) can achieve combination synchronization-II or combination synchronization-I with the response system (7).

Corollary 11. (i) Let $\beta_{1}=\beta_{2}=\beta_{3}=1, \gamma_{1}=\gamma_{2}=\gamma_{3}=0$, $\delta_{1}=\delta_{2}=\delta_{3}=0, \varepsilon_{1}=\varepsilon_{2}=\varepsilon_{3}=0$, and $\eta_{1}=\eta_{2}=\eta_{3}=0$; if the controllers are chosen as

$$
u=\left(\begin{array}{c}
-f_{1} \\
-\left(\frac{\left(a_{4}-1\right)\left(c_{4}+1\right)}{a_{4}}+a_{4}-\alpha_{3} x_{3}\right) v_{11}-\left(c_{4}-a_{4}+2\right) v_{12}-g_{1} \\
\frac{1-a_{4}}{a_{4}} v_{11}\left(v_{11}+\alpha_{1} x_{1}\right)-\alpha_{2} x_{2} v_{11}+\left(b_{4}-1\right) v_{13}-h_{1}
\end{array}\right),
$$

the drive system (4) will achieve projective synchronization with the response system (7). (ii) Let $\alpha_{1}=\alpha_{2}=\alpha_{3}=0, \beta_{1}=\beta_{2}=\beta_{3}=0, \delta_{1}=\delta_{2}=$ $\delta_{3}=1, \varepsilon_{1}=\varepsilon_{2}=\varepsilon_{3}=0$, and $\eta_{1}=\eta_{2}=\eta_{3}=0$; if the controllers are chosen as

$$
u=\left(\begin{array}{c}
-f_{2} \\
-\left(\frac{\left(a_{4}-1\right)\left(c_{4}+1\right)}{a_{4}}+a_{4}-\gamma_{3} y_{3}\right) v_{21}-\left(c_{4}-a_{4}+2\right) v_{22}-g_{2} \\
\frac{1-a_{4}}{a_{4}} v_{21}\left(v_{21}+\gamma_{1} y_{1}\right)-\gamma_{2} y_{2} v_{21}+\left(b_{4}-1\right) v_{23}-h_{2}
\end{array}\right),
$$


the drive system (5) will achieve projective synchronization with the response system (7). (iii) Let $\alpha_{1}=\alpha_{2}=\alpha_{3}=0, \beta_{1}=\beta_{2}=\beta_{3}=0, \gamma_{1}=\gamma_{2}=$ $\gamma_{3}=0, \delta_{1}=\delta_{2}=\delta_{3}=0$, and $\eta_{1}=\eta_{2}=\eta_{3}=1$; if the controllers are chosen as

$$
u=\left(\begin{array}{c}
-f_{3} \\
-\left(\frac{\left(a_{4}-1\right)\left(c_{4}+1\right)}{a_{4}}+a_{4}-\varepsilon_{3} w_{3}\right) v_{31}-\left(c_{4}-a_{4}+2\right) v_{32}-g_{3} \\
\frac{1-a_{4}}{a_{4}} v_{31}\left(v_{31}+\varepsilon_{1} w_{1}\right)-\varepsilon_{2} w_{2} v_{31}+\left(b_{4}-1\right) v_{33}-h_{3}
\end{array}\right),
$$

the drive system (6) will achieve projective synchronization with the response system (7).

Corollary 12. Let $\alpha_{1}=\alpha_{2}=\alpha_{3}=0, \gamma_{1}=\gamma_{2}=\gamma_{3}=0$, $\varepsilon_{1}=\varepsilon_{2}=\varepsilon_{3}=0$, and (i) $\beta_{1}=\beta_{2}=\beta_{3}=1, \delta_{1}=\delta_{2}=\delta_{3}=0$, $\eta_{1}=\eta_{2}=\eta_{3}=0$, or (ii) $\beta_{1}=\beta_{2}=\beta_{3}=0, \delta_{1}=\delta_{2}=\delta_{3}=1$, $\eta_{1}=\eta_{2}=\eta_{3}=0$, or (iii) $\beta_{1}=\beta_{2}=\beta_{3}=0, \delta_{1}=\delta_{2}=\delta_{3}=0$, $\eta_{1}=\eta_{2}=\eta_{3}=1$; if the controllers are chosen as

$u$

$$
=\left(\begin{array}{c}
0 \\
-\left(\frac{\left(a_{4}-1\right)\left(c_{4}+1\right)}{a_{4}}+a_{4}\right) v_{1}-\left(c_{4}-a_{4}+2\right) v_{2} \\
\frac{1-a_{4}}{a_{4}} v_{1}^{2}+\left(b_{4}-1\right) v_{3}
\end{array}\right),
$$

in which $v_{1}=z_{1}, v_{2}=z_{2}-\left(a_{4}-1\right) z_{1} / a_{4}, v_{3}=z_{3}$, the equilibrium $(0,0,0)$ of the response system (7) is globally asymptotically stable.

Numerical simulations are performed to illustrate the above combination synchronization-II among systems (4), (5), (6), and (7). Fourth-order Runge-Kutta method is applied with time step equal to 0.001 , and the initial values of variables $x, y, w$, and $z$ are provided randomly. We choose $\alpha_{i}=\beta_{i}=$ $\gamma_{i}=\delta_{i}=\eta_{i}=1, \varepsilon_{i}=3(i=1,2,3)$. The parameter values of systems (4), (5), (6), and (7) are the same as described along with the systems, i.e., $a_{1}=10, b_{1}=28, c_{1}=8 / 3, a_{2}=35$, $b_{2}=3, c_{2}=28, a_{3}=0.2, b_{3}=0.2, c_{3}=5.7, a_{4}=36, b_{4}=3$, and $c_{4}=20$. With these given conditions, the controller can be calculated as

$$
\begin{aligned}
u_{1}= & 26 s_{1}\left(x_{1}-x_{2}\right)+s_{2}\left(y_{1}-y_{2}\right) \\
& +s_{3}\left(108 w_{1}-111 w_{2}-3 w_{3}\right), \\
u_{2}= & -s_{1}\left(56+\frac{5}{12}-x_{3}\right) e_{11}-s_{2}\left(56+\frac{5}{12}-y_{3}\right) e_{21} \\
& -s_{3}\left(56+\frac{5}{12}-3 w_{3}\right) e_{31} \\
& +14 s_{1}\left(e_{12}-\frac{35}{36} e_{11}\right)+14 s_{2}\left(e_{22}-\frac{35}{36} e_{21}\right) \\
& +14 s_{3}\left(e_{32}-\frac{35}{36} e_{31}\right)-7 s_{1}\left(3 x_{2}-4 x_{1}\right)
\end{aligned}
$$

$$
\begin{aligned}
& -s_{2}\left(7 y_{1}+12 y_{2}\right) \\
& -3 s_{3}\left(19.8 w_{2}-3 w_{1} w_{3}-w_{1}\right), \\
u_{3}= & -s_{1} e_{11}\left(\frac{35}{36}\left(e_{11}+x_{1}\right)+x_{2}\right) \\
& -s_{2} e_{21}\left(\frac{35}{36}\left(e_{21}+y_{1}\right)+y_{2}\right) \\
& -s_{3} e_{31}\left(\frac{35}{36}\left(e_{31}+3 w_{1}\right)+3 w_{2}\right) \\
& +2\left(s_{1} e_{13}+s_{2} e_{23}+s_{3} e_{33}\right)+\frac{1}{3} s_{1} x_{3} \\
& -3 s_{3}\left(3 w_{1} w_{2}-w_{1} w_{3}+2.7 w_{3}-0.2\right) .
\end{aligned}
$$

Substituting the equations of controller into the error system, we then have (notice that $s_{1}=1, s_{2}=0$, and $s_{3}=0$ for the dynamics of $e_{11}, e_{12}$, and $e_{13}, s_{1}=0, s_{2}=1$, and $s_{3}=0$ for the dynamics of $e_{21}, e_{22}$, and $e_{23}, s_{1}=0, s_{2}=0$, and $s_{3}=1$ for the dynamics of $e_{31}, e_{32}$, and $e_{33}$ ),

$$
\begin{aligned}
& \dot{e}_{11}=36\left(e_{12}-e_{11}\right) \\
& \dot{e}_{12}=34 e_{12}-\left(70+\frac{1}{36}\right) e_{11}-e_{11} e_{13}-x_{1} e_{13}, \\
& \dot{e}_{13}=e_{11} e_{12}+x_{1} e_{12}-e_{13}-\frac{35}{36} e_{11}\left(e_{11}+x_{1}\right), \\
& \dot{e}_{21}=36\left(e_{22}-e_{21}\right), \\
& \dot{e}_{22}=34 e_{22}-\left(70+\frac{1}{36}\right) e_{21}-e_{21} e_{23}-y_{1} e_{23}, \\
& \dot{e}_{23}=e_{21} e_{22}+y_{1} e_{22}-e_{23}-\frac{35}{36} e_{21}\left(e_{21}+y_{1}\right), \\
& \dot{e}_{31}=36\left(e_{32}-e_{31}\right), \\
& \dot{e}_{32}=34 e_{32}-\left(70+\frac{1}{36}\right) e_{31}-e_{31} e_{33}-3 w_{1} e_{33}, \\
& \dot{e}_{33}=e_{31} e_{32}+3 w_{1} e_{32}-e_{33}-\frac{35}{36} e_{31}\left(e_{31}+3 w_{1}\right) .
\end{aligned}
$$

Via numerical calculations on the above error system, Figure 2 demonstrates the time response of the synchronization errors $e_{i j}(i=1,2,3$ and $j=1,2,3)$, which converges 

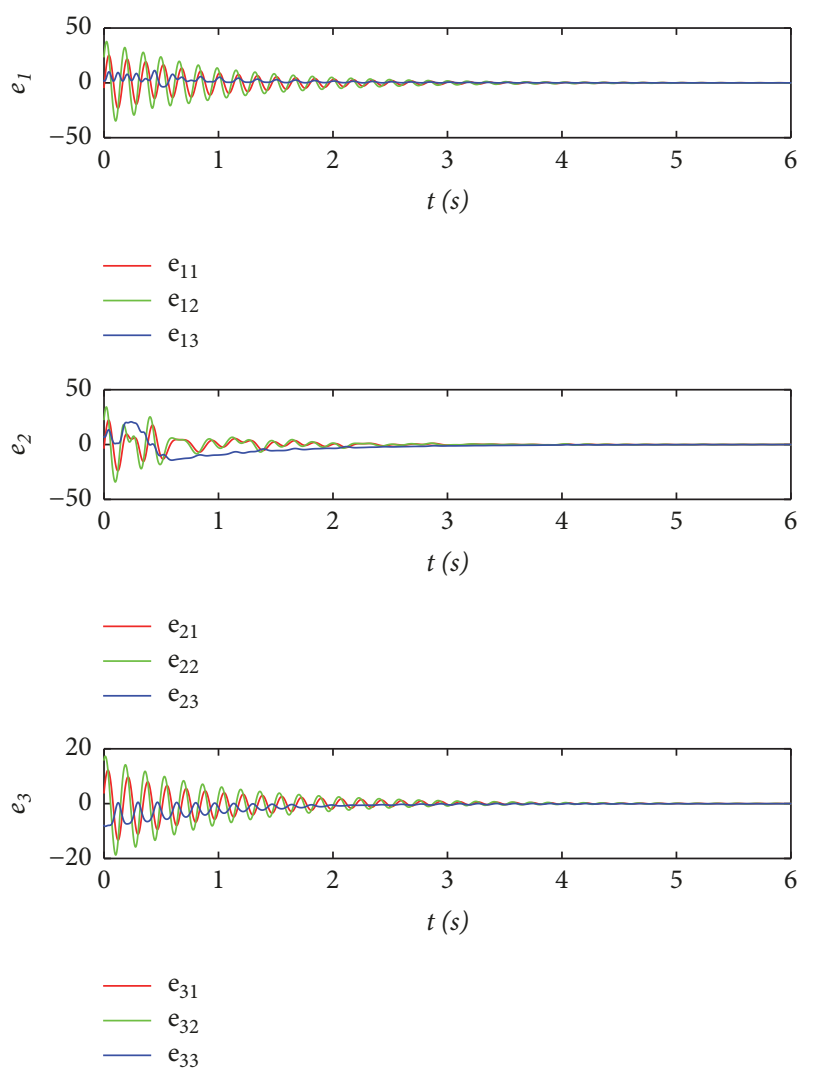

FIGURE 2: Time response of synchronization errors $e_{i j}, i=1,2,3$ and $j=1,2,3$, for the combination synchronization-II of Case 1 among systems (4), (5), (6), and (7).

to zero, suggesting that the combination synchronizationII among systems (4), (5), (6), and (7) indeed achieves. Figure 3 depicts time response of the states, $x_{i}, y_{i}, w_{i}$, and $z_{i}$, showing the synchronization between the drive systems and the response system.

\section{Combination Synchronization-II of Case 2}

In this section, the combination synchronization-II of Case 2 among the four chaotic systems, Lorenz system, Chen system, Rössler system, and Lü system, is realized. Lorenz system, Chen system, and Rössler system are also taken as drive systems, in which Lorenz system and Chen system are on the same branch as shown in Figure 1(c); Lü system is taken as the response system.

According to Definition 1, the combination synchronization of Case 2 among the four chaotic systems can be described by

$$
\lim _{t \longrightarrow+\infty}\|A x+B y-C z\|+\lim _{t \longrightarrow+\infty}\|D w-E z\|=0 .
$$

Let

$$
\begin{aligned}
& e_{1}=C z-A x-B y, \\
& e_{2}=E z-D w,
\end{aligned}
$$

and then the error system can be described by

$$
\dot{e}_{11}=\frac{a_{4} \gamma_{1}}{\gamma_{2}} e_{12}-a_{4} e_{11}+f_{1}+\gamma_{1} u_{1}
$$

$$
\begin{aligned}
\dot{e}_{12} & =c_{4} e_{12}-\frac{\gamma_{2}}{\gamma_{1} \gamma_{3}}\left(e_{11} e_{13}+\left(\alpha_{3} x_{3}+\beta_{3} y_{3}\right) e_{11}\right. \\
& \left.+\left(\alpha_{1} x_{1}+\beta_{1} y_{1}\right) e_{13}\right)+g_{1}+\gamma_{2} u_{2}, \\
\dot{e}_{13} & =\frac{\gamma_{3}}{\gamma_{1} \gamma_{2}}\left(e_{11} e_{12}+\left(\alpha_{2} x_{2}+\beta_{2} y_{2}\right) e_{11}\right. \\
& \left.+\left(\alpha_{1} x_{1}+\beta_{1} y_{1}\right) e_{12}\right)-b_{4} e_{13}+h_{1}+\gamma_{3} u_{3}, \\
\dot{e}_{21} & =\frac{a_{4} \varepsilon_{1}}{\varepsilon_{2}} e_{22}-a_{4} e_{21}+f_{2}+\varepsilon_{1} u_{1}, \\
\dot{e}_{22} & =c_{4} e_{22}-\frac{\varepsilon_{2}}{\varepsilon_{1} \varepsilon_{3}} e_{21} e_{23}-\frac{\delta_{3} \varepsilon_{2}}{\varepsilon_{1} \varepsilon_{3}} w_{3} e_{21}-\frac{\delta_{1} \varepsilon_{2}}{\varepsilon_{1} \varepsilon_{3}} w_{1} e_{23} \\
& +g_{2}+\varepsilon_{2} u_{2}, \\
\dot{e}_{23} & =\frac{\varepsilon_{3}}{\varepsilon_{1} \varepsilon_{2}} e_{21} e_{22}+\frac{\delta_{2} \varepsilon_{3}}{\varepsilon_{1} \varepsilon_{2}} w_{2} e_{21}+\frac{\delta_{1} \varepsilon_{3}}{\varepsilon_{1} \varepsilon_{2}} w_{1} e_{22}-b_{4} e_{23} \\
& +h_{2}+\varepsilon_{3} u_{3},
\end{aligned}
$$

in which

$$
\begin{aligned}
f_{1}= & \frac{a_{4} \alpha_{2} \gamma_{1}}{\gamma_{2}} x_{2}+\frac{a_{4} \beta_{2} \gamma_{1}}{\gamma_{2}} y_{2}-a_{4}\left(\alpha_{1} x_{1}+\beta_{1} y_{1}\right) \\
& -a_{1} \alpha_{1}\left(x_{2}-x_{1}\right)-a_{2} \beta_{1}\left(y_{2}-y_{1}\right), \\
g_{1}= & -b_{1} \alpha_{2} x_{1}+\left(c_{4}+1\right) \alpha_{2} x_{2}+\left(a_{2}-c_{2}\right) \beta_{2} y_{1} \\
& +\left(c_{4}-c_{2}\right) \beta_{2} y_{2}+\alpha_{2} x_{1} x_{3}+\beta_{2} y_{1} y_{3} \\
& -\frac{\gamma_{2}}{\gamma_{1} \gamma_{3}}\left(\alpha_{1} x_{1}+\beta_{1} y_{1}\right)\left(\alpha_{3} x_{3}+\beta_{3} y_{3}\right), \\
h_{1}= & \left(c_{1}-b_{4}\right) \alpha_{3} x_{3}+\left(b_{2}-b_{4}\right) \beta_{2} y_{3} \\
& +\frac{\gamma_{3}}{\gamma_{1} \gamma_{2}}\left(\alpha_{1} x_{1}+\beta_{1} y_{1}\right)\left(\alpha_{2} x_{2}+\beta_{2} y_{2}\right) \\
& -\alpha_{3} x_{1} x_{2}-\beta_{3} y_{1} y_{2}, \\
f_{2}= & -a_{4} \delta_{1} w_{1}+\left(\frac{a_{4} \delta_{2} \varepsilon_{1}}{\varepsilon_{2}}+\delta_{1}\right) w_{2}+\delta_{1} w_{3}, \\
g_{2}= & -\frac{\delta_{1} \delta_{3} \varepsilon_{2}}{\varepsilon_{1} \varepsilon_{3}} w_{1} w_{3}-\delta_{2} w_{1}+\left(c_{4}-a_{3}\right) \delta_{2} w_{2}, \\
h_{2}= & \frac{\delta_{1} \delta_{2} \varepsilon_{3}}{\varepsilon_{1} \varepsilon_{2}} w_{1} w_{2}-\delta_{3} w_{1} w_{3}+\left(c_{3}-b_{4}\right) \delta_{3} w_{3}-b_{3} \delta_{3} .
\end{aligned}
$$

The active backstepping design method is also applied for designing the controllers $u_{1}, u_{2}$, and $u_{3}$ for the error system (34).

Theorem 13. If the controllers $u_{1}, u_{2}$, and $u_{3}$ are designed as

$$
\begin{aligned}
& u_{1}=-\frac{s_{1}}{\gamma_{1}} f_{1}-\frac{s_{2}}{\varepsilon_{1}} f_{2}, \\
& u_{2}=-s_{1}\left(\frac{\left(a_{4}-1\right)\left(c_{4}+1\right)}{a_{4} \gamma_{1}}+\frac{a_{4} \gamma_{1}}{\gamma_{2}^{2}}-\frac{\alpha_{3} x_{3}+\beta_{3} y_{3}}{\gamma_{1} \gamma_{3}}\right)
\end{aligned}
$$



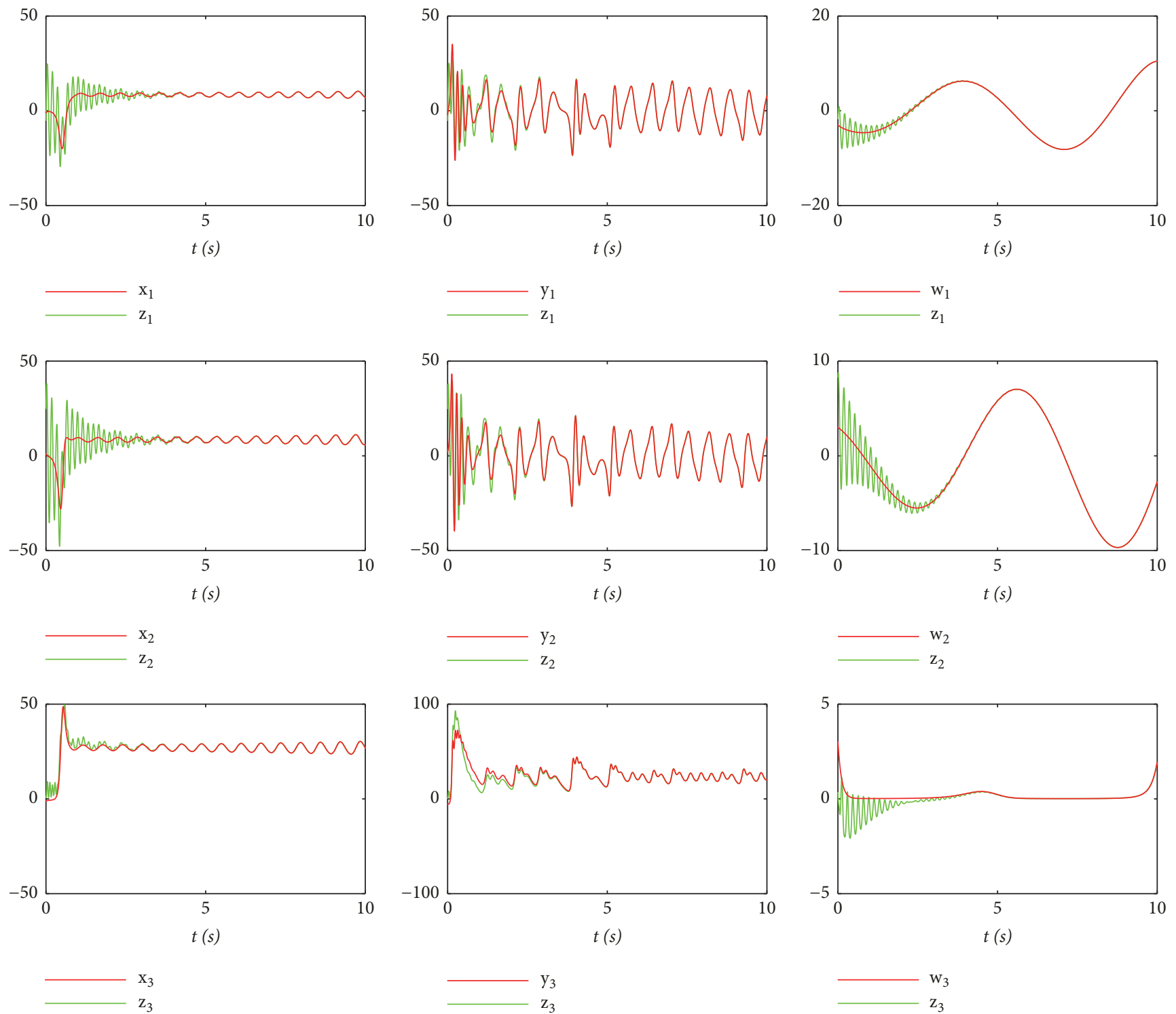

FIgURE 3: Time response of states $x_{i}, y_{i}, w_{i}$, and $z_{i}, i=1,2,3$, and the comparison of states between the drive systems and the response system.

$$
\begin{aligned}
& \cdot v_{11}-\left(c_{4}-a_{4}+2\right)\left(\frac{s_{1}}{\gamma_{2}} v_{12}+\frac{s_{2}}{\varepsilon_{2}} v_{22}\right) \\
& \left.+\alpha_{1} x_{1}+\beta_{1} y_{1}\right) v_{12}+\left(b_{4}-1\right)\left(\frac{s_{1}}{\gamma_{3}} v_{13}+\frac{s_{2}}{\varepsilon_{3}} v_{23}\right) \\
& -s_{2}\left(\frac{\left(a_{4}-1\right)\left(c_{4}+1\right)}{a_{4} \varepsilon_{1}}+\frac{a_{4} \varepsilon_{1}}{\varepsilon_{2}^{2}}-\frac{\delta_{3} w_{3}}{\varepsilon_{1} \varepsilon_{3}}\right) v_{21}-\frac{s_{1}}{\gamma_{2}} \\
& -\frac{s_{1}}{\gamma_{3}} h_{1}-\frac{s_{2}}{\varepsilon_{3}} h_{2} \\
& \text { - } g_{1}-\frac{s_{2}}{\varepsilon_{2}} g_{2} \text {, } \\
& u_{3}=-s_{1}\left[\frac{a_{4}-1}{a_{4} \gamma_{1}^{2}} v_{11}^{2}\right. \\
& +\frac{1}{\gamma_{1}}\left(\frac{\alpha_{2} x_{2}+\beta_{2} y_{2}}{\gamma_{2}}+\frac{\left(a_{4}-1\right)\left(\alpha_{1} x_{1}+\beta_{1} y_{1}\right)}{a_{4} \gamma_{1}}\right) \\
& \begin{array}{l}
s_{1}=\operatorname{sgn}(\|x\|+\|y\|)(1-\operatorname{sgn}(\|w\|)), \\
s_{2}=\operatorname{sgn}(\|w\|)(1-\operatorname{sgn}(\|x\|+\|y\|)),
\end{array} \\
& \begin{array}{l}
s_{1}=\operatorname{sgn}(\|x\|+\|y\|)(1-\operatorname{sgn}(\|w\|)), \\
s_{2}=\operatorname{sgn}(\|w\|)(1-\operatorname{sgn}(\|x\|+\|y\|)),
\end{array} \\
& \left.\cdot v_{11}\right]-s_{2}\left[\left(\frac{a_{4}-1}{a_{4} \varepsilon_{1}^{2}} v_{21}+\frac{1}{\varepsilon_{1}}\left(\frac{1}{\varepsilon_{2}}-\frac{\varepsilon_{2}}{\varepsilon_{3}^{2}}\right) v_{22}\right)\right. \\
& \left.\cdot\left(v_{21}+\delta_{1} w_{1}\right)+\frac{\delta_{2}}{\varepsilon_{1} \varepsilon_{2}} w_{2} v_{21}\right]-\frac{s_{1}}{\gamma_{1}}\left(\frac{1}{\gamma_{2}}-\frac{\gamma_{2}}{\gamma_{3}^{2}}\right)\left(v_{11}\right. \\
& \left(\begin{array}{c}
v_{11} \\
v_{12} \\
v_{13}
\end{array}\right)=\left(\begin{array}{ccc}
1 & 0 & 0 \\
\frac{\left(1-a_{4}\right) \gamma_{2}}{a_{4} \gamma_{1}} & 1 & 0 \\
0 & 0 & 1
\end{array}\right)\left(\begin{array}{l}
e_{11} \\
e_{12} \\
e_{13}
\end{array}\right) \text {, }
\end{aligned}
$$




$$
\left(\begin{array}{l}
v_{21} \\
v_{22} \\
v_{23}
\end{array}\right)=\left(\begin{array}{ccc}
1 & 0 & 0 \\
\frac{\left(1-a_{4}\right) \varepsilon_{2}}{a_{4} \varepsilon_{1}} & 1 & 0 \\
0 & 0 & 1
\end{array}\right)\left(\begin{array}{l}
e_{21} \\
e_{22} \\
e_{23}
\end{array}\right)
$$

the combination synchronization-II of Case 2 will be achieved among systems (4), (5), (6), and (7).

Proof. Based on the linear transformation described in (38), we construct $\left(v_{11}, v_{12}, v_{13}\right)$-subsystem. For this subsystem, we have $\|w\|=0$. Then direct calculations lead to

$$
\begin{aligned}
\dot{v}_{11} & =\frac{a_{4} \gamma_{1}}{\gamma_{2}}\left(v_{12}-\frac{\left(1-a_{4}\right) \gamma_{2}}{a_{4} \gamma_{1}} v_{11}\right)-a_{4} v_{11}+f_{1} \\
& +\left.\gamma_{1} u_{1}\right|_{\|x\|+\|y\|>0,\|w\|=0}=-v_{11}+\frac{a_{4} \gamma_{1}}{\gamma_{2}} v_{12}, \\
\dot{v}_{12} & =\frac{\left(1-a_{4}\right) \gamma_{2}}{a_{4} \gamma_{1}}\left(-v_{11}+\frac{a_{4} \gamma_{1}}{\gamma_{2}} v_{12}\right)+c_{4}\left(v_{12}\right. \\
& \left.-\frac{\left(1-a_{4}\right) \gamma_{2}}{a_{4} \gamma_{1}} v_{11}\right)-\frac{\gamma_{2}}{\gamma_{1} \gamma_{3}}\left(v_{11} v_{13}\right. \\
& \left.+\left(\alpha_{3} x_{3}+\beta_{3} y_{3}\right) v_{11}+\left(\alpha_{1} x_{1}+\beta_{1} y_{1}\right) v_{13}\right)+g_{1} \\
& +\left.\gamma_{2} u_{2}\right|_{\|x\|+\|y\|>0,\|w\|=0}=-\frac{\gamma_{2}}{\gamma_{1} \gamma_{3}}\left(v_{11}+\alpha_{1} x_{1}\right. \\
& \left.+\beta_{1} y_{1}\right) v_{13}-\frac{a_{4} \gamma_{1}}{\gamma_{2}} v_{11}-v_{12}, \\
\dot{v}_{13} & =\frac{\gamma_{3}}{\gamma_{1} \gamma_{2}}\left(\left(\alpha_{2} x_{2}+\beta_{2} y_{2}\right) v_{11}\right. \\
& \left.+\left(v_{11}+\alpha_{1} x_{1}+\beta_{1} y_{1}\right)\left(v_{12}-\frac{\left(1-a_{4}\right) \gamma_{2}}{a_{4} \gamma_{1}} v_{11}\right)\right) \\
& -b_{4} v_{13}+h_{1}+\left.\gamma_{3} u_{3}\right|_{\|x\|+\|y\|>0,\|w\|=0}=\frac{\gamma_{2}}{\gamma_{1} \gamma_{3}}\left(v_{11}\right. \\
& \left.+\alpha_{1} x_{1}+\beta_{1} y_{1}\right) v_{12}-v_{13} .
\end{aligned}
$$

Take a Lyapunov function as

$$
V_{1}=\frac{1}{2} v_{11}^{2}+\frac{1}{2} v_{12}^{2}+\frac{1}{2} v_{13}^{2}
$$

for the $\left(v_{11}, v_{12}, v_{13}\right)$-subsystem. The derivative of $V_{1}$ is given by

$$
\begin{aligned}
\dot{V}_{1} & =v_{11} \dot{v}_{11}+v_{12} \dot{v}_{12}+v_{13} \dot{v}_{13}=v_{11}\left(-v_{11}+\frac{a_{4} \gamma_{1}}{\gamma_{2}} v_{12}\right) \\
& +v_{12}\left(-\frac{\gamma_{2}}{\gamma_{1} \gamma_{3}}\left(v_{11}+\alpha_{1} x_{1}+\beta_{1} y_{1}\right) v_{13}-\frac{a_{4} \gamma_{1}}{\gamma_{2}} v_{11}\right. \\
& \left.-v_{12}\right)+v_{13}\left(\frac{\gamma_{2}}{\gamma_{1} \gamma_{3}}\left(v_{11}+\alpha_{1} x_{1}+\beta_{1} y_{1}\right) v_{12}-v_{13}\right) \\
& =-v_{11}^{2}-v_{12}^{2}-v_{13}^{2} \leq 0,
\end{aligned}
$$

which suggests that the equilibrium $(0,0,0)$ is globally asymptotically stable for the $\left(v_{11}, v_{12}, v_{13}\right)$-subsystem. Likewise, we construct $\left(v_{21}, v_{22}, v_{23}\right)$-subsystem which satisfies $\|x\|+\|y\|=0$. Then we have

$$
\begin{aligned}
\dot{v}_{21}= & \frac{a_{4} \varepsilon_{1}}{\varepsilon_{2}}\left(v_{22}+\frac{\left(a_{4}-1\right) \varepsilon_{2}}{a_{4} \varepsilon_{1}} v_{21}\right)-a_{4} v_{21}+f_{2} \\
& +\left.\varepsilon_{1} u_{1}\right|_{\|x\|+\|y\|=0,\|w\|>0}=-v_{21}+\frac{a_{4} \varepsilon_{1}}{\varepsilon_{2}} v_{22}, \\
\dot{v}_{22}= & \frac{\left(1-a_{4}\right) \varepsilon_{2}}{a_{4} \varepsilon_{1}}\left(-v_{21}+\frac{a_{4} \varepsilon_{1}}{\varepsilon_{2}} v_{22}\right) \\
& +c_{4}\left(v_{22}+\frac{\left(a_{4}-1\right) \varepsilon_{2}}{a_{4} \varepsilon_{1}} v_{21}\right)-\frac{\varepsilon_{2}}{\varepsilon_{1} \varepsilon_{3}} v_{21} v_{23} \\
& -\frac{\delta_{3} \varepsilon_{2}}{\varepsilon_{1} \varepsilon_{3}} w_{3} v_{21}-\frac{\delta_{1} \varepsilon_{2}}{\varepsilon_{1} \varepsilon_{3}} w_{1} v_{23}+g_{2} \\
& +\left.\varepsilon_{2} u_{2}\right|_{\|x\|+\|y\|=0,\|w\|>0} \\
= & -\frac{a_{4} \varepsilon_{1}}{\varepsilon_{2}} v_{21}-v_{22}-\frac{\varepsilon_{2}}{\varepsilon_{1} \varepsilon_{3}}\left(v_{21}+\delta_{1} w_{1}\right) v_{23}, \\
\dot{v}_{23}= & \left(\frac{\varepsilon_{3}}{\varepsilon_{1} \varepsilon_{2}} v_{21}+\frac{\delta_{1} \varepsilon_{3}}{\varepsilon_{1} \varepsilon_{2}} w_{1}\right)\left(v_{22}+\frac{\left(a_{4}-1\right) \varepsilon_{2}}{a_{4} \varepsilon_{1}} v_{21}\right) \\
& +\frac{\delta_{2} \varepsilon_{3}}{\varepsilon_{1} \varepsilon_{2}} w_{2} v_{21}-b_{4} v_{23}+h_{2}+\left.\varepsilon_{3} u_{3}\right|_{\|x\|+\|y\|=0,\|w\|>0} \\
= & \frac{\varepsilon_{2}}{\varepsilon_{1} \varepsilon_{3}}\left(v_{21}+\delta_{1} w_{1}\right) v_{22}-v_{23} .
\end{aligned}
$$

Choosing a candidate Lyapunov function,

$$
V_{2}=\frac{1}{2} v_{21}^{2}+\frac{1}{2} v_{22}^{2}+\frac{1}{2} v_{23}^{2},
$$

for the $\left(v_{21}, v_{22}, v_{23}\right)$-subsystem. The derivative of $V_{2}$ is given by

$$
\begin{aligned}
\dot{V}_{2}= & v_{21} \dot{v}_{21}+v_{22} \dot{v}_{22}+v_{23} \dot{v}_{23} \\
= & v_{21}\left(-v_{21}+\frac{a_{4} \varepsilon_{1}}{\varepsilon_{2}} v_{22}\right) \\
& +v_{22}\left(-\frac{a_{4} \varepsilon_{1}}{\varepsilon_{2}} v_{21}-v_{22}-\frac{\varepsilon_{2}}{\varepsilon_{1} \varepsilon_{3}}\left(v_{21}+\delta_{1} w_{1}\right) v_{23}\right) \\
& +v_{23}\left(\frac{\varepsilon_{2}}{\varepsilon_{1} \varepsilon_{3}}\left(v_{21}+\delta_{1} w_{1}\right) v_{22}-v_{23}\right) \\
= & -v_{21}^{2}-v_{22}^{2}-v_{23}^{2} \leq 0,
\end{aligned}
$$

which suggests that $\left(v_{21}, v_{22}, v_{23}\right)$ asymptotically approaches $(0,0,0)$.

Considering the above results together, we have $\left(v_{11}, v_{12}, v_{13}\right) \longrightarrow(0,0,0)$ and $\left(v_{21}, v_{22}, v_{23}\right) \longrightarrow(0,0,0)$ as $t \longrightarrow+\infty$. Via the linear transformation described by (38), $e_{i j} \longrightarrow 0(i=1,2 ; j=1,2,3)$ is obtained. It means the establishment of (32). 
The following corollary of combination synchronizationI between three drive systems and one response system can be directly obtained from Theorem 13 . For the other cases, one can refer to Corollaries 9 12.

Corollary 14. If the controllers are chosen as

$u$

$$
=\left(\begin{array}{l}
\frac{\left(\left.\gamma_{1} u_{1}\right|_{\|x\|+\|y\|>0,\|w\|=0}+\left.\varepsilon_{1} u_{1}\right|_{\|x\|+\|y\|=0,\|w\|>0}\right)}{\left(\gamma_{1}+\varepsilon_{1}\right)} \\
\frac{\left(\left.\gamma_{2} u_{2}\right|_{\|x\|+\|y\|>0,\|w\|=0}+\left.\varepsilon_{2} u_{2}\right|_{\|x\|+\|y\|=0,\|w\|>0}\right)}{\left(\gamma_{2}+\varepsilon_{2}\right)} \\
\frac{\left(\left.\gamma_{3} u_{3}\right|_{\|x\|+\|y\|>0,\|w\|=0}+\left.\varepsilon_{3} u_{3}\right|_{\|x\|+\|y\|=0,\|w\|>0}\right)}{\left(\gamma_{3}+\varepsilon_{3}\right)}
\end{array}\right),
$$

in which $u_{1}, u_{2}$, and $u_{3}$ are described by (36), we will have

$$
\lim _{t \longrightarrow+\infty}\|A x+B y+D w-(C+E) z\|=0 .
$$

Hence, the drive systems (4), (5), and (6) can achieve combination synchronization-I with the response system (7).

For demonstrating the second case of combination synchronization-II, an example of numerical simulation is provided in the following. The parameter values for the combination synchronization-II and the dynamics of the four chaotic systems are given as $\alpha_{i}=\beta_{i}=\gamma_{i}=\delta_{i}=\eta_{i}=\varepsilon_{i}=1$ $(i=1,2,3), a_{1}=10, b_{1}=28, c_{1}=8 / 3, a_{2}=35, b_{2}=3$, $c_{2}=28, a_{3}=0.2, b_{3}=0.2, c_{3}=5.7, a_{4}=36, b_{4}=3$, and $c_{4}=20$. Then based on the above theoretical calculations, the controller can be determined as

$$
\begin{aligned}
u_{1}= & s_{1}\left(26 x_{1}-26 x_{2}+y_{1}-y_{2}\right) \\
& +s_{2}\left(36 w_{1}-37 w_{2}-w_{3}\right), \\
u_{2}= & -s_{1}\left(56+\frac{5}{12}-x_{3}-y_{3}\right) e_{11} \\
& +14\left(s_{1}\left(e_{12}-\frac{35}{36} e_{11}\right)+s_{2}\left(e_{22}-\frac{35}{36} e_{21}\right)\right) \\
& -s_{2}\left(56+\frac{5}{12}-w_{3}\right) e_{21} \\
& +s_{1}\left(28 x_{1}-21 x_{2}-7 y_{1}+8 y_{2}+x_{1} y_{3}+x_{3} y_{1}\right) \\
& +s_{2}\left(w_{1} w_{3}+w_{1}-19.8 w_{2}\right), \\
u_{3}= & -s_{1}\left[\frac{35}{36} e_{11}^{2}+\left(x_{2}+y_{2}+\frac{35}{36}\left(x_{1}+y_{1}\right)\right) e_{11}\right] \\
& -s_{2}\left[\frac{35}{36} e_{21}\left(e_{21}+w_{1}\right)+w_{2} e_{21}\right] \\
& +2\left(s_{1} e_{13}+s_{2} e_{23}\right) \\
& +s_{1}\left(\frac{1}{3} x_{3}-x_{1} y_{2}-x_{2} y_{1}\right) \\
& -s_{2}\left(w_{1} w_{2}-w_{1} w_{3}+2.7 w_{3}-0.2\right) .
\end{aligned}
$$
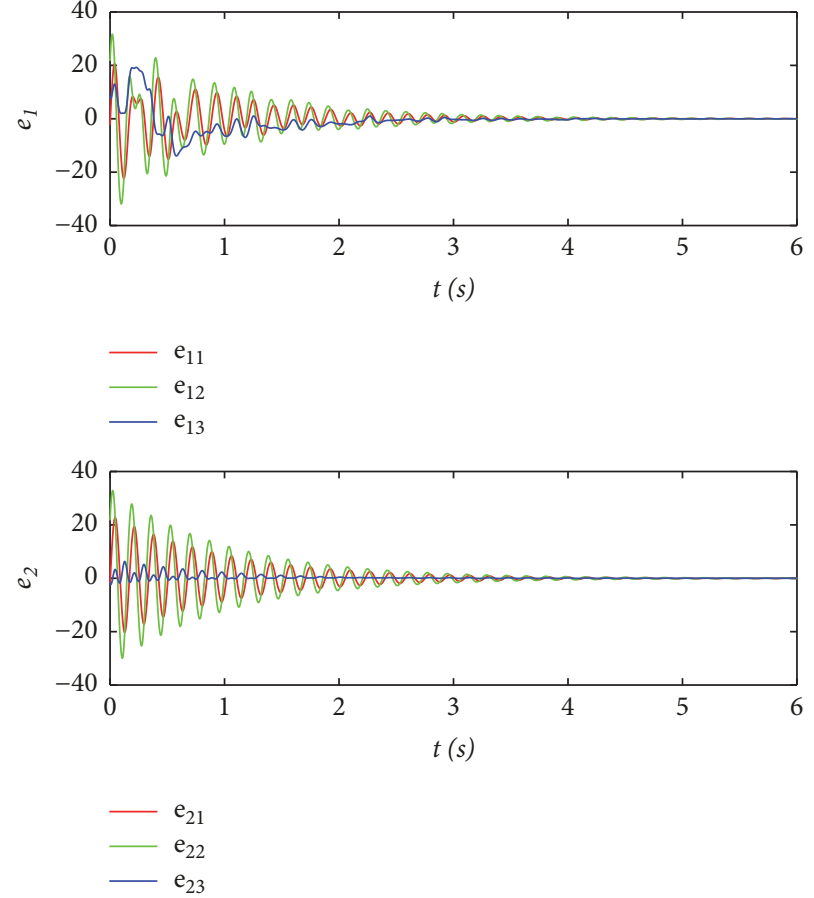

FIGURE 4: Time response of synchronization errors $e_{i j}, i=1,2$ and $j=1,2,3$, for the combination synchronization-II of Case 2 among systems (4), (5), (6), and (7).

With this controller, the error system can be changed as the following (notice that $s_{1}=1$ and $s_{2}=0$ for the dynamics of $e_{11}$, $e_{12}$, and $e_{13}, s_{1}=0$ and $s_{2}=1$ for the dynamics of $e_{21}, e_{22}$, and $\left.e_{23}\right)$ :

$$
\begin{aligned}
\dot{e}_{11}= & 36\left(e_{12}-e_{11}\right), \\
\dot{e}_{12}= & 34 e_{12}-\left(70+\frac{1}{36}\right) e_{11}-e_{11} e_{13}-\left(x_{1}+y_{1}\right) e_{13}, \\
\dot{e}_{13}= & e_{11} e_{12}-e_{13}-\frac{35}{36} e_{11}^{2}+\left(x_{1}+y_{1}\right) e_{12} \\
& -\frac{35}{36}\left(x_{1}+y_{1}\right) e_{11}, \\
\dot{e}_{21}= & 36\left(e_{22}-e_{21}\right), \\
\dot{e}_{22}= & 34 e_{22}+\left(43-\frac{7}{36}\right) e_{21}-e_{21} e_{23}-w_{1} e_{23}, \\
\dot{e}_{23}= & e_{21} e_{22}-\frac{35}{36} e_{21} e_{21}+w_{1} e_{22}-\frac{35}{36} w_{1} e_{21}-e_{23} .
\end{aligned}
$$

By numerically solving the above error system, Figure 4 is plotted. Figure 4 shows that the synchronization errors $e_{i j}$ $(i=1,2$ and $j=1,2,3)$ approach zero as time increases. This suggests that the combination synchronization-II of Case 2 as described above is realized. The time response of states $x_{i}+y_{i}$, $w_{i}$, and $z_{i}$ as shown in Figure 5 and their comparison further prove that. 

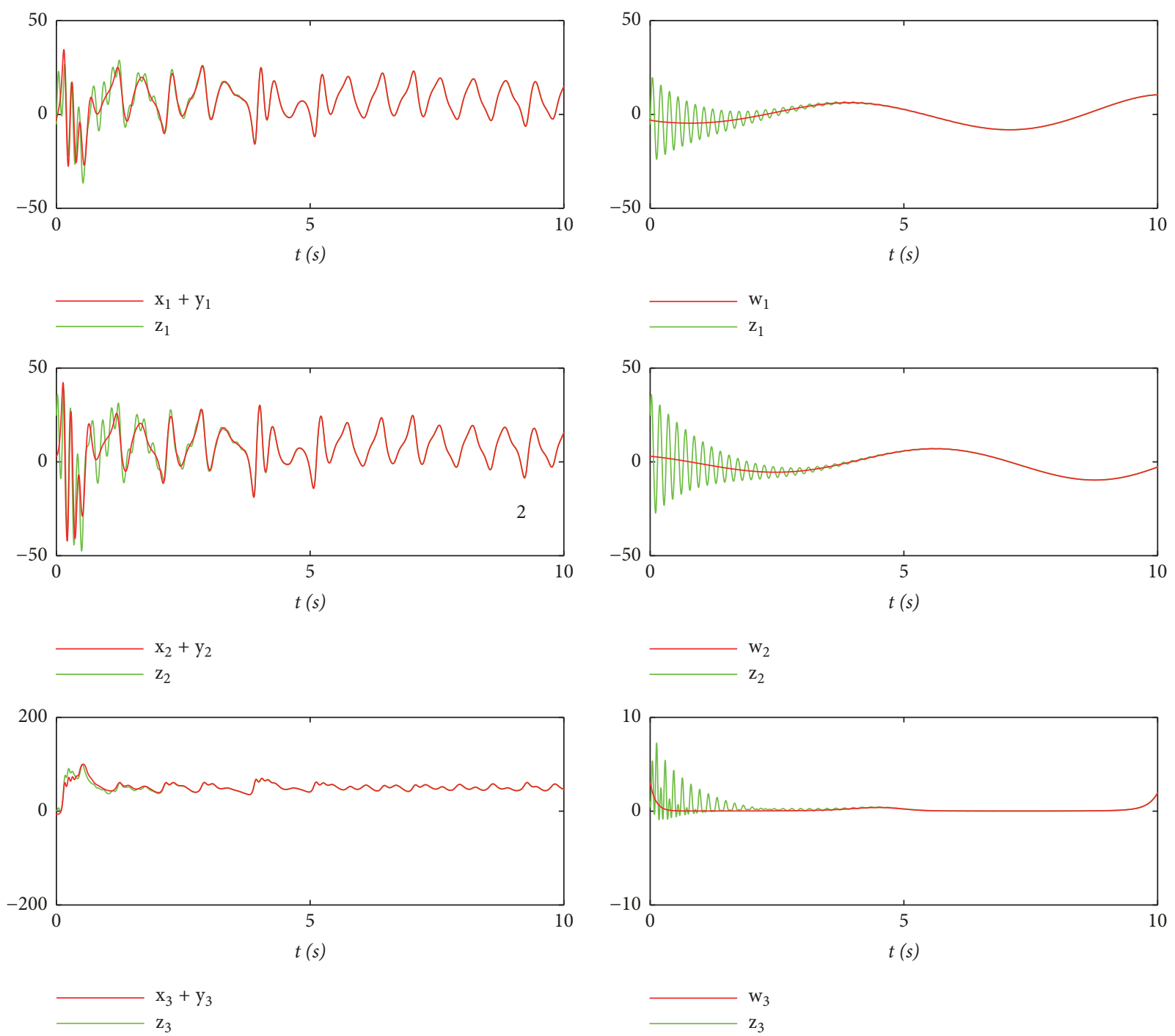

FIGURE 5: Comparison of states between $x_{i}+y_{i}$ and $z_{i}$, and $w_{i}$ and $z_{i}, i=1,2,3$.

\section{Discussion and Conclusions}

This research develops a new type of combination synchronization, which is referred as combination synchronizationII. Based on the active backstepping design method, the combination synchronization-II between three drive systems and one response system is achieved. Numerical simulations are provided to show the feasibility and effectiveness of the proposed combination synchronization approach, with applications in four classical chaotic systems. Moreover, it should be noticed that such application can be extended to other chaotic systems. For example, when multiple memristor systems of infinite chaotic attractors stay at different chaotic states, the combination synchronization-II can take these memristor chaotic systems into the same oscillation.

The proposed combination synchronization approach develops the synchronization techniques among multiple chaotic systems. On one hand, the proposed approach potentially has wider applications than former combination synchronizations. On the other hand, the proposed approach can be applied for more complex system combinations. Via comparison with previous approaches, the following advantages of combination synchronization-II can be emphasized:

(1) The combination synchronization-II is more general than the combination synchronization-I or combination complex synchronization. Based on the norm properties and the conditions of combination synchronization-II, the sufficient conditions for the combination synchronization-I or the combination complex synchronization can be deduced.

(2) The combination synchronization-II can be applied for a variety of system combination modes than the combination synchronization-I or the combination complex synchronization. This shows advantages in practical applications. For example, in secure communication when several signals are transmitted, diversified modes of system combinations can effectively increase antitranslated capability. 
(3) Based on the proposed approach, an applicable universal combination synchronization scheme can be designed for multiple chaotic systems which feature series and/or parallel system combinations. This enables wider potential applications of the combination synchronization approach in many fields.

\section{Data Availability}

The data of numerical results are generated during the study.

\section{Conflicts of Interest}

The authors declare that they have no conflicts of interest.

\section{Acknowledgments}

This research is supported by the National Natural Science Foundation of China (NNSFC) through grant No. 11602234 and talent scientific research fund of LSHU (No. 2017XJJ058).

\section{References}

[1] S. Boccaletti, J. Kurths, G. Osipov, D. L. Valladares, and C. S. Zhou, "The synchronization of chaotic systems," Physics Reports, vol. 366, no. 1-2, pp. 1-101, 2002.

[2] L. M. Pecora and T. L. Carroll, "Synchronization in chaotic systems," Controlling Chaos, vol. 6, no. 8, pp. 142-145, 1996.

[3] B. Blasius and L. Stone, "Chaos and phase synchronization in ecological systems," International Journal of Bifurcation and Chaos, vol. 10, no. 10, pp. 2361-2380, 2000.

[4] H.-K. Chen, "Global chaos synchronization of new chaotic systems via nonlinear control," Chaos, Solitons \& Fractals, vol. 23, no. 4, pp. 1245-1251, 2005.

[5] R. K. Upadhyay and V. Rai, "Complex dynamics and synchronization in two non-identical chaotic ecological systems," Chaos, Solitons \& Fractals, vol. 40, no. 5, pp. 2233-2241, 2009.

[6] J. Q. Lu and J. D. Cao, "Adaptive complete synchronization of two identical or different chaotic (hyperchaotic) systems with fully unknown parameters," Chaos: An Interdisciplinary Journal of Nonlinear Science, vol. 15, no. 4, p. 043901, 2005.

[7] C. Yao, Q. Zhao, and J. Yu, "Complete synchronization induced by disorder in coupled chaotic lattices," Physics Letters A, vol. 377, no. 5, pp. 370-377, 2013.

[8] M. G. Rosenblum, A. S. Pikovsky, and J. Kurths, "Phase synchronization of chaotic oscillators," Physical Review Letters, vol. 76, no. 11, pp. 1804-1807, 1996.

[9] A. Pikovsky, M. Rosenblum, and J. Kurths, "Phase synchronization in regular and chaotic systems," International Journal of Bifurcation and Chaos, vol. 10, no. 10, pp. 2291-2305, 2000.

[10] Y.-W. Wang and Z.-H. Guan, "Generalized synchronization of continuous chaotic system," Chaos, Solitons \& Fractals, vol. 27, no. 1, pp. 97-101, 2006.

[11] Z. Q. Yang, Q. Zhang, and Z. Q. Chen, "Adaptive linear generalized synchronization between two nonidentical networks," Communications in Nonlinear Science and Numerical Simulation, vol. 17, no. 6, pp. 2628-2636, 2012.
[12] L. Runzi, W. Yinglan, and D. Shucheng, "Combination synchronization of three classic chaotic systems using active backstepping design," Chaos: An Interdisciplinary Journal of Nonlinear Science, vol. 21, no. 4, p. 043114, 2011.

[13] J. Sun, Y. Shen, G. Zhang, C. Xu, and G. Cui, "Combinationcombination synchronization among four identical or different chaotic systems," Nonlinear Dynamics, vol. 73, no. 3, pp. 12111222, 2013.

[14] R. Luo and Y. Wang, "Finite-time stochastic combination synchronization of three different chaotic systems and its application in secure communication," Chaos: An Interdisciplinary Journal of Nonlinear Science, vol. 22, no. 2, p. 023109, 2012.

[15] Z. Wu and X. Fu, "Combination synchronization of three different order nonlinear systems using active backstepping design," Nonlinear Dynamics, vol. 73, no. 3, pp. 1863-1872, 2013.

[16] K. S. Ojo, A. N. Njah, O. I. Olusola, and M. . Omeike, "Generalized reduced-order hybrid combination synchronization of three Josephson junctions via backstepping technique," Nonlinear Dynamics, vol. 77, no. 3, pp. 583-595, 2014.

[17] J. Sun, G. Cui, Y. Wang, and Y. Shen, "Combination complex synchronization of three chaotic complex systems," Nonlinear Dynamics, vol. 79, no. 2, pp. 953-965, 2015.

[18] U. E. Vincent, A. O. Saseyi, and P. V. McClintock, "Multiswitching combination synchronization of chaotic systems," Nonlinear Dynamics, vol. 80, no. 1-2, pp. 845-854, 2015.

[19] H. Xi, Y. Li, and X. Huang, "Adaptive function projective combination synchronization of three different fractional-order chaotic systems," Optik - International Journal for Light and Electron Optics, vol. 126, no. 24, pp. 5346-5349, 2015.

[20] S. B. Wang, X. Y. Wang, X. Y. Wang, and Y. F. Zhou, "Adaptive generalized combination complex synchronization of uncertain real and complex nonlinear systems," AIP Advances, vol. 6, no. 4, p. 045011, 2016.

[21] I. Ahmad, M. Shafiq, and M. M. Al-Sawalha, "Globally exponential multi switching-combination synchronization control of chaotic systems for secure communications," Chinese Journal of Physics, vol. 56, no. 3, pp. 974-987, 2018.

[22] A. Khan and S. Singh, "Chaotic analysis and combinationcombination synchronization of a novel hyperchaotic system without any equilibria," Chinese Journal of Physics, vol. 56, no. 1, pp. 238-251, 2018.

[23] G. M. Mahmoud, T. M. Abed-Elhameed, and M. E. Ahmed, "Generalization of combination-combination synchronization of chaotic n-dimensional fractional-order dynamical systems," Nonlinear Dynamics, vol. 83, no. 4, pp. 1885-1893, 2016.

[24] J. Sun, Y. Wang, G. Cui, and Y. Shen, "Dynamical properties and combination-combination complex synchronization of four novel chaotic complex systems," Optik - International Journal for Light and Electron Optics, vol. 127, no. 4, pp. 1572-1580, 2016.

[25] K. S. Ojo, A. N. Njah, and O. I. Olusola, "Compoundcombination synchronization of chaos in identical and different orders chaotic systems," Archives of Control Sciences. Polish Academy of Sciences. Committee of Automatic Control and Robotics, vol. 25(61), no. 4, pp. 463-490, 2015.

[26] Z. Alam, L. Yuan, and Q. Yang, "Chaos and combination synchronization of a new fractional-order system with two stable node-foci," IEEE/CAA Journal of Automatica Sinica, vol. 3, no. 2, pp. 157-164, 2016.

[27] A. Khan and Shikha, "Combination synchronization of Genesio time delay chaotic system via robust adaptive sliding mode control," International Journal of Dynamics and Control, vol. 6, no. 2, pp. 758-767, 2018. 
[28] J. Sun, Y. Wang, Y. Wang, and Y. Shen, "Finite-time synchronization between two complex-variable chaotic systems with unknown parameters via nonsingular terminal sliding mode control," Nonlinear Dynamics, vol. 85, no. 2, pp. 1105-1117, 2016.

[29] J. Sun, Y. Wu, G. Cui, and Y. Wang, "Finite-time real combination synchronization of three complex-variable chaotic systems with unknown parameters via sliding mode control," Nonlinear Dynamics, vol. 88, no. 3, pp. 1677-1690, 2017.

[30] A. K. Singh, V. K. Yadav, and S. Das, "Dual combination synchronization of the fractional order complex chaotic systems," Journal of Computational and Nonlinear Dynamics, vol. 12, no. 1, pp. 011017-0110178, 2017.

[31] J. Gebhardt and R. Kruse, "Parallel combination of information sources," in Belief Change, vol. 3, pp. 393-439, Springer, Dordrecht, Netherlands, 1998.

[32] M. J. Brennan, A. Carrella, T. P. Waters, and V. Lopes Jr., "On the dynamic behaviour of a mass supported by a parallel combination of a spring and an elastically connected damper," Journal of Sound and Vibration, vol. 309, no. 3-5, pp. 823-837, 2008.

[33] T. Yang, C.-M. Yang, and L.-B. Yang, "Control of Rössler system to periodic motions using impulsive control methods," Physics Letters A, vol. 232, no. 5, pp. 356-361, 1997.

[34] G. A. Leonov and N. V. Kuznetsov, "On differences and similarities in the analysis of Lorenz, Chen, and LU systems," Applied Mathematics and Computation, vol. 256, pp. 334-343, 2015.

[35] F. Zhang and Y. Shu, "Global dynamics for the simplified Lorenz system model," Applied Mathematics and Computation, vol. 259, pp. 53-60, 2015.

[36] J. Sun, X. Zhao, J. Fang, and Y. Wang, "Autonomous memristor chaotic systems of infinite chaotic attractors and circuitry realization," Nonlinear Dynamics, vol. 94, no. 4, pp. 2879-2887, 2018.

[37] J. Sun, G. Han, Y. Wang, H. Zhang, and L. Wu, "Hybrid memristor chaotic system," Journal of Nanoelectronics and Optoelectronics, vol. 13, no. 6, pp. 812-818, 2018. 


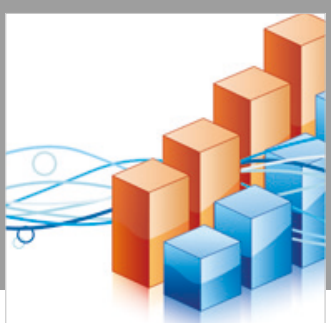

Advances in

Operations Research

\section{-n-m}
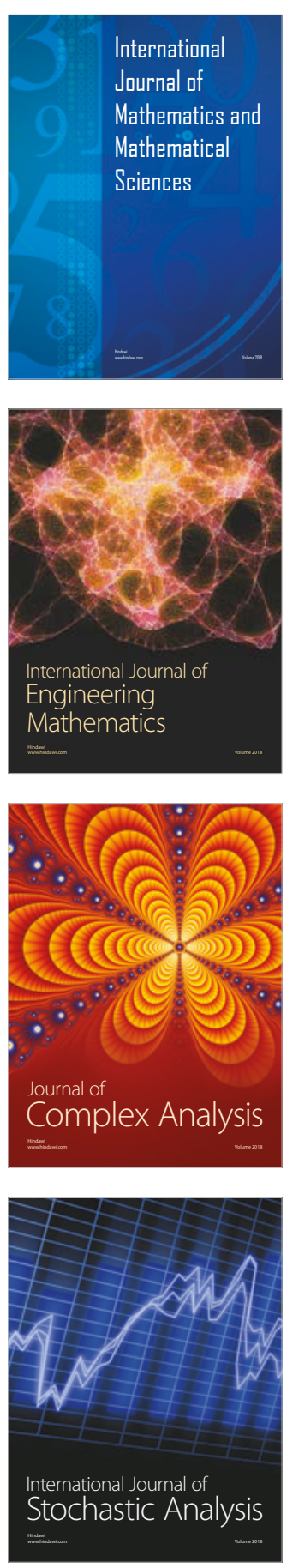
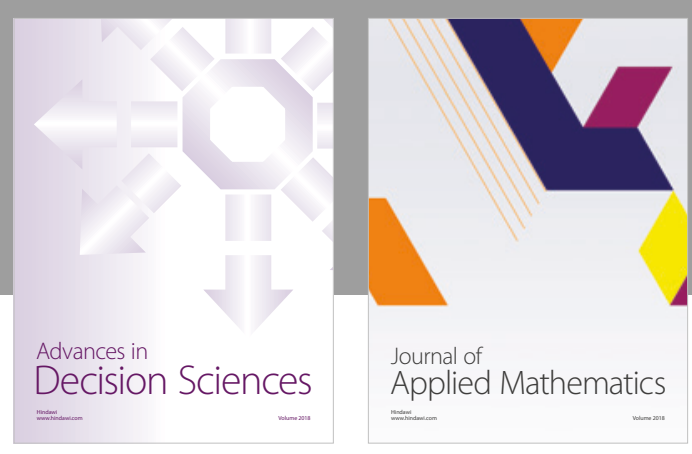

Journal of

Applied Mathematics
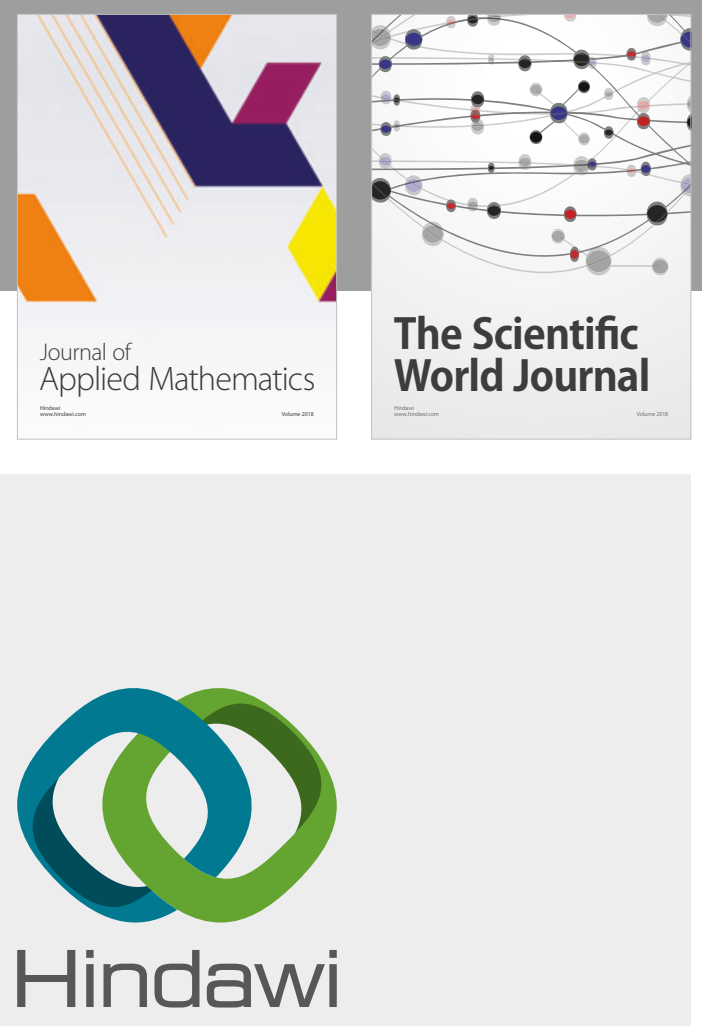

Submit your manuscripts at

www.hindawi.com

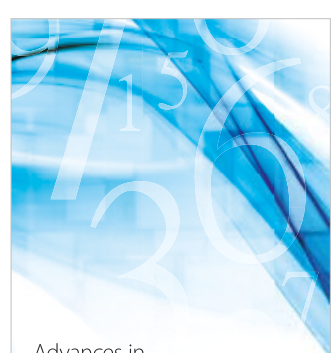

Advances in
Numerical Analysis
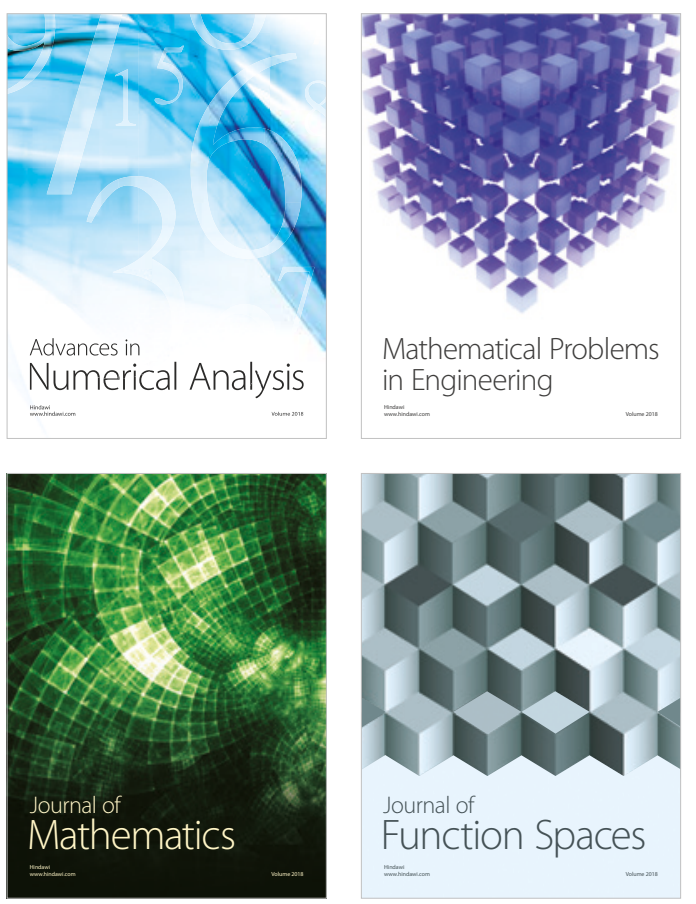

Mathematical Problems in Engineering

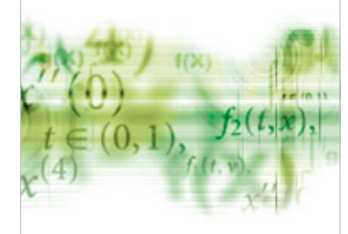

International Journal of

Differential Equations

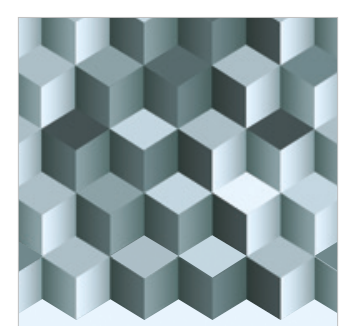

Journal of

Function Spaces

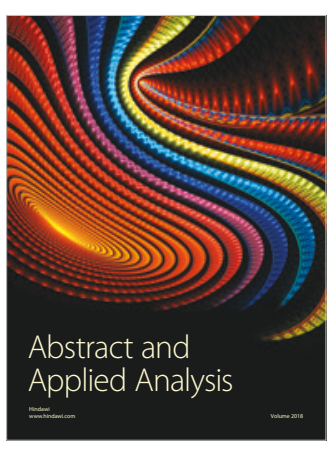

The Scientific

World Journal

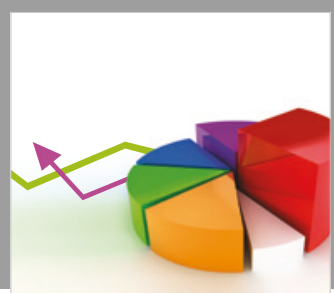

Journal of

Probability and Statistics
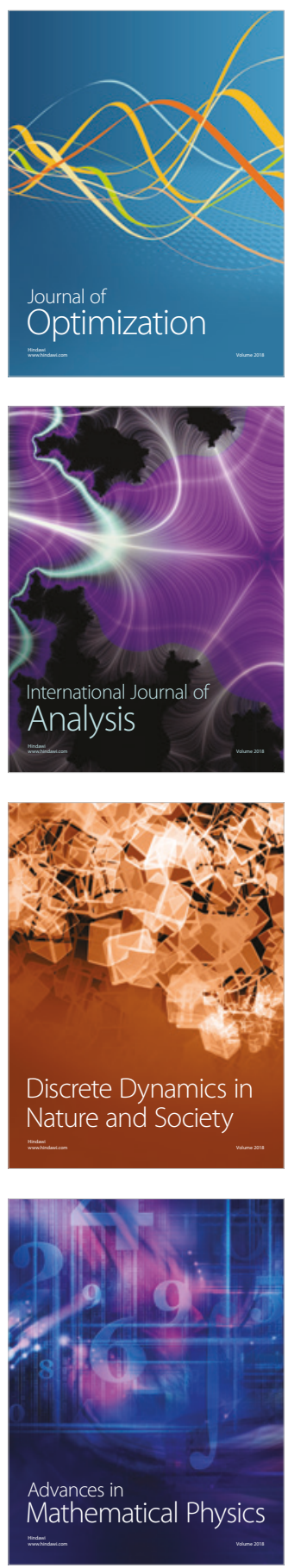